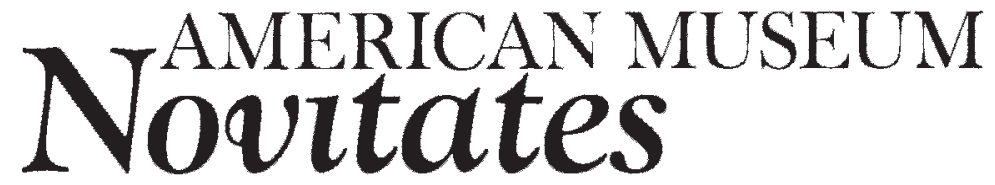

PUBLISHED BY THE AMERICAN MUSEUM OF NATURAL HISTORY CENTRAL PARK WEST AT 79TH STREET, NEW YORK, NY 10024 Number 3593, 31 pp., 12 figures

December 12, 2007

\title{
Synopsis of the Eastern North American Species of the Plant Bug Genus Parthenicus, with Descriptions of Three New Species and a Revised Key (Heteroptera: Miridae: Orthotylinae)
}

\author{
THOMAS J. HENRY ${ }^{1}$
}

\begin{abstract}
Three new species of the orthotyline genus Parthenicus Reuter are described and the seven previously known species of eastern North America are diagnosed. Parthenicus cruentus, n.sp. is described from Nebraska, $P$. sedumicola, n.sp. from Arkansas, and P. wheeleri, n.sp. from Oklahoma and Texas. A lectotype from Texas is designated and male genitalia are illustrated for $P$. psalliodes Reuter, the type species of the genus. Also provided are selected scanning electron photomicrographs and illustrations of the male genitalia for new species, a color habitus illustration for $P$. sedumicola, and for all species a color dorsal habitus photograph, hosts, and distribution information. A revised identification key to the 10 eastern species is given to help distinguish species.
\end{abstract}

\section{INTRODUCTION}

The plant bug genus Parthenicus Reuter is a group of small, slender, often sexually dimorphic, orthotyline mirids currently numbering 80 species (Henry and Wheeler, 1988; Schuh, 1995, Schwartz and Scudder, 2003). Most occur in the United States (73 species), with only one species known from Jamaica, one from Guatemala, and two from Baja California, Mexico. Seven species co-occur in Canada and the United States, with only $P$. brooksi Kelton, P. lalannei Schwartz and Scudder, and $P$. thibodeaui Schwartz and Scudder being unique to Canada (Henry and Wheeler, 1988; Schwartz and Scudder, 2003). Parthenicus nigrosquamus Maldonado, previously recorded from Puerto Rico, was trans-

\footnotetext{
${ }^{1}$ Systematic Entomology Laboratory, PSI, ARS, USDA, c/o P.O. Box 37012, National Musuem of Natural History, MRC-0168, Smithsonian Institution, Washington, DC 20013-7012 (thenry@sel.barc.usda.gov).
} 
ferred to the genus Proboscidotylus Henry and reported for the first time in the United States from Key West, Florida (Henry, 2003); Knight (1968) described 36 new species and provided a key to the western North American species; Henry (1982) reviewed and gave a key to the seven eastern species, including three new ones; and Schwartz and Scudder (2003) added two new species for Canada.

The genus is in great need of revision. Many species have been described from one or a few poor specimens, usually without host information, making them difficult to identify, and numerous new species remain to be described in the western United States, Mexico, Central America, and the West Indies.

In this paper I describe three new eastern United States species to provide names for Dr. A.G. Wheeler, who is studying their hosts and biology. Herein, I diagnose the seven known eastern North American species, designate a lectotype for $P$. psalliodes Reuter, the type species of the genus, and describe one new species from Arkansas, one from Nebraska, and one from Oklahoma and Texas. Color dorsal habitus photographs for each species, a color dorsal illustration of $P$. sedumicola, selected scanning electron photomicrographs, illustrations of the male genitalia for the new species and P. psalliodes, hosts and distributions, and a revised key for the eastern North American species are given.

Depositories for specimens cited in this paper are as follows: American Museum of Natural History, New York, NY (AMNH); Canadian National Collection of Insects, Ottawa, Ontario (CNC); and National Museum of Natural History, Washington, DC (USNM).

Plant names used in this paper follow the National PLANTS Database (USDA, NRCS 2004).

\section{PARTHENICUS REUTER}

Parthenicus Reuter, 1876: 84 (orig. descrip.), Kirkaldy, 1906: 128 (list); Carvalho, 1958: 122 (cat.), Knight, 1968: 129 (n. spp., key western spp.); Henry, 1982: 355 (descrip., key eastern spp.); Henry and Wheeler, 1988: 436 (cat.); Schuh, 1995: 177 (cat.). Type species: Parthenicus psalliodes Reuter. Monotypic.
Diagnosis: Species of Parthenicus are distinguished by the small size, dull to weakly shining, impunctate, dorsal surface, the absence of a basal carina on the head, the sericeous and scalelike setae on the dorsum, and the saltatorial hindlegs. The enlarged or swollen hindfemora give species in this genus the capability to jump, a trait that is especially apparent in brachypterous females and nymphs.

Description: Small, length $4.5 \mathrm{~mm}$ or less (eastern U.S. spp. $3.5 \mathrm{~mm}$ or less), elongate oval, impunctate; head without a distinct carina, eyes large, strongly granulate (more so in males); labium extending to metacoxae or beyond; pubescence simple, intermixed with golden, silvery, and/or black sericeous to scalelike setae, with black, scalelike setae especially abundant on cuneus and apical area of corium; hemelytra subparallel; males macropterous with the hemelytra and membrane well developed; females macropterous or brachypterous, membrane and cuneus often strongly abbreviated or coleopteriform, with the claval suture absent, forming a beetlelike "elytron"; hindfemora strongly saltatorial; and parempodia fleshy and convergent. Vesica enclosed in a prominent phallothecal sheath and usually bearing one or two simple spiculi; the left paramere usually C-shaped, with base thickened and the arch of the $\mathrm{C}$ gradually tapered; and the right paramere ranging from straight, thickened, and spined distally to slender and arching or C-shaped.

Discussion: Considerable variation in color and markings of some species makes study of male genitalia essential for positive identification of species. The parameres, though relatively simple compared with many orthotylines, are distinctive for most species (Knight, 1964; Henry 1982; Scudder and Schwartz, 2003). The left paramere (figs. 25, $28,56,74)$ is somewhat C-shaped, with the distal neck becoming much more slender than the base. The right paramere is comprised of a stout stem, usually with an apical spine or tubercle (figs. 57, 75), often with an acute lateral process or serrate ridge (fig. 29), or may be more slender, arching, and C-shaped (fig. 26). The phallotheca (figs. 27, 30, 59, 77) is attached to the phallobase, but it protrudes well into the genital capsule opening and is 
visible behind the parameres when view caudally. This is unusual for most genera of Orthotylini I have studied, and is analogous only with members of the tribe Ceratocapsini and at least some Austromirini.

An analysis of relationships within Parthenicus is beyond the scope of this paper and must await a comprehensive generic revision. There are, however, clear indications of species groups based on male genitalia, even within the eastern species. The parameres are very homogeneous among most of the eastern species (Henry, 1982). The left paramere groups the species $P$. cruentus, $P$. juniperi, $P$. knighti, $P$. psalliodes, $P$. rufus, $P$. taxodii, $P$. vaccini, and $P$. weemsi, and the right paramere groups these same species, with the exception of $P$. cruentus and $P$. vaccini. Interestingly, the eastern species grouped by parameres also are the ones having only fully macropterous females. Parthenicus cruentus, P. sedumicola, $P$. vaccini, and $P$. wheeleri all have some form of brachyptery in females, a trait commonly seen in species from arid habitats, including pine barrens of the East where $P$. vaccini is found (Henry, 1978; Roble and Hoffman, 2000).

The relationship of Parthenicus with other Orthotylinae also is unknown. Schuh (1974) considered Parthenicus to have typical Orthotylus-type male genitalia, with welldeveloped, long, heavily schlerotized spiculi on the vesica. My dissections confirm that each eastern species has at least one, and often two, simple spiculi (figs. 27, 30), one of which may be relatively elaborate (fig. 76), with multiple processes. As noted above, of all Orthotylini I have examined, Parthenicus is the only genus having the phallotheca visible externally within the genital capsule opening, a condition not too unlike that found in the Austromirini and Ceratocapsini.

\section{KEY TO THE SPECIES OF PARTHENICUS IN EASTERN UNITED STATES}

1. Tibial spines without distinct spots at bases or, at most, with a few vague spots on the basal third of the hindtibia ........... 2

- Tibial spines with distinct red or brown spots at bases on all tibiae ............ . 6
2. Metafemora strongly infuscated; dorsum with black scalelike setae. . . . . . . . . . 3

- Metafemora uniformly pale yellow or testaceous, never fuscous; dorsum without black scalelike setae ............. 5

3. Pronotum and hemelytra usually pale to yellowish brown, lacking red spots, but usually tinged with red or salmon pink, especially on cuneus; scutellum predominantly fuscous; hindfemora reddish brown to almost fuscous, except for a narrow pale area along ventral surface; black scalelike setae concentrated on scutellum and apical one-third of corium; both males and females macropterous; distribution widespread in the eastern United States; hosts Juniperus spp. and other Cupressaceae . . . . . . . . . . .

juniperi (Heidemann)

- Pronotum and hemelytra pale, with scattered red to reddish-brown spots; scutellum pale, sometimes weakly infuscated basally, but always with apex pale; hindfemora uniformly red to reddish brown on distal two-thirds, never pale ventrally; black scalelike setae more evenly scattered on hemelytra or nearly absent; male macropterous, known females strongly brachypterous. . . . . . . . . 4

4. Antennal segment $I$ in males longer than width of vertex between eyes, in females subequal to width of vertex; left paramere (fig. 56), right paramere (fig. 57); distribution Arkansas; host Sedum sp. . . . . . sedumicola, n.sp.

- Antennal segment I in males and females less than width of vertex between eyes; left paramere (fig. 74), right paramere (fig. 75); distribution Oklahoma and Texas; hosts selected species of Poaceae. . wheeleri, n.sp.

5. Dorsum uniformly yellow, without red or fuscous markings; hemelytral membrane smoky gray; rostrum short, just reaching middle of metacoxae; distribution Maryland to Florida, west to Missouri; host Taxodium distichum. . . . . . . . . taxodii Knight

- Dorsum yellow to testaceous, with the base and outer margin of the clavus and base of cuneus accented with red or small red spots, and basal angles of scutellum weakly infuscated; hemelytral membrane uniformly fuscous to black; rostrum long, extending beyond metacoxae; distribution coastal Florida; host unknown ........... knighti Henry

6. Dorsum and ventral surface predominantly deep red to reddish orange, with only a few, small, irregular pale areas showing through red................. 7

- Dorsum and ventral surface pale grayish, testaceous, or pale brownish to reddish orange, not predominantly red or reddish 


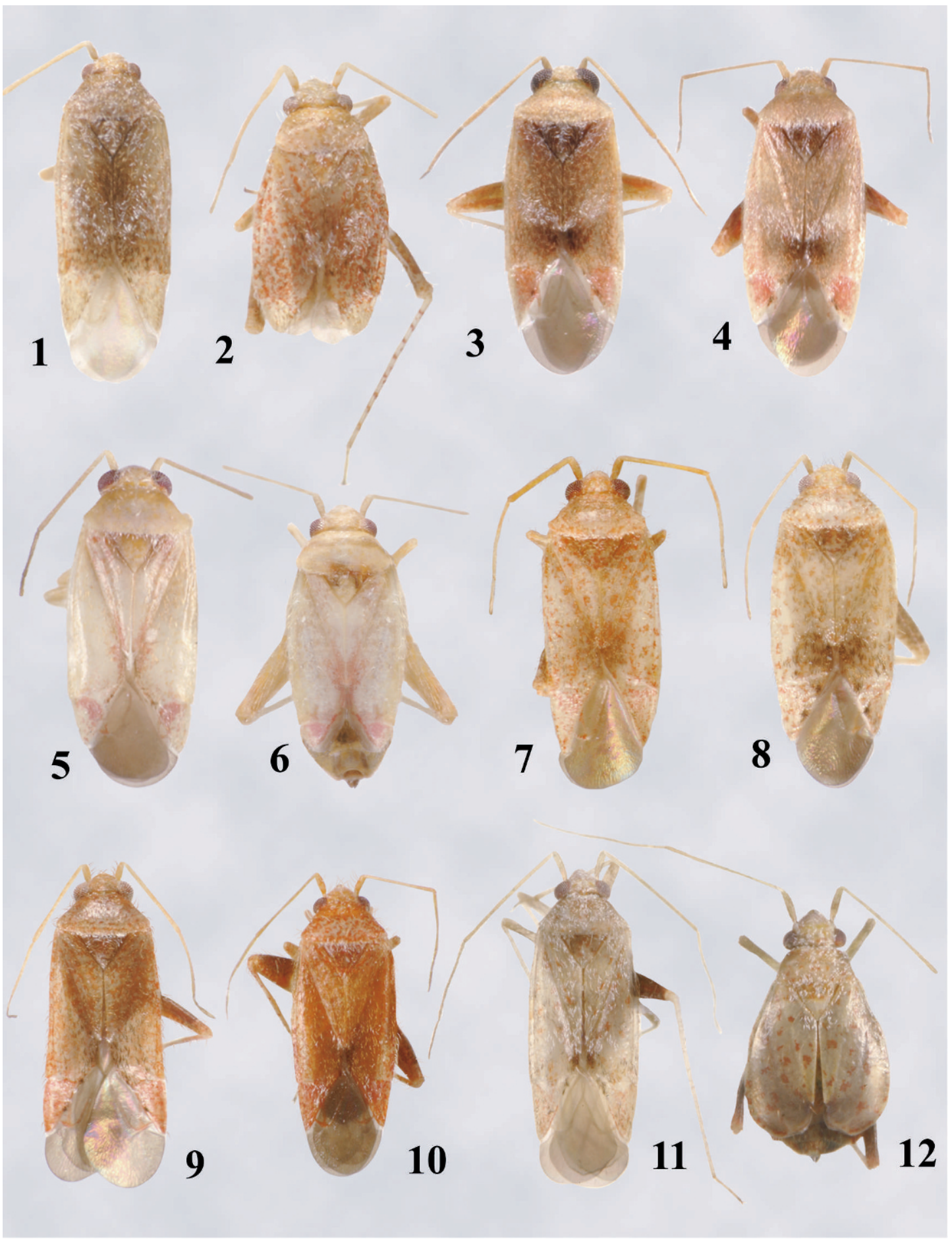

Figs. 1-12. Habitus figures of Parthenicus spp. 1, P. cruentus, male (Nebraska, Johnson Co., nr. Vesta, 11 Jul 1986). 2, P. cruentus, female (same data as for male). 3, P. juniperi, male (Maryland, Montgomery Co., 
orange, with or without red or brown spots .............. 8

7. Corium and clavus uniformly deep red to reddish orange with only a few fine, irregular, pale areas; white scalelike setae concentrated narrowly along posterior pronotal margin, basal half of scutellum, and apex of clavus; spots at bases of tibial spines small, red, often indistinct or diffused; distribution Florida, Georgia, and North Carolina; hosts Hypericum spp. . . . . . . . rufus Henry

- Corium deep red to reddish orange, clavus largely pale with only a few small, scattered, red spots; pronotal disk, scutellum, and clavus densely clothed with white scalelike setae; spots at bases of tibial spines distinct and deep red to dark brown; distribution Florida; host Ceratiola ericoides . . . . . . . . .......... weemsi Henry (dark form)

8. Dorsum, first and second antennal segments, and legs pale gray or grayish green, with numerous brown to fuscous spots; wing membrane white with two fuscous clouds apically; females usually brachypterous; distribution eastern coast, west to Michigan in the north; host Hudsonia spp. and Lechia maritima......... vaccini (Van Duzee)

- Dorsum testaceous or pale brownish to reddish orange, with numerous to only a few red spots on dorsum ............ 9

9. Dorsum pale brownish or reddish orange, with only a few brown or red spots at base and apex of corium, spots on legs red; antennae uniformly pale testaceous, without spots; wing membrane smoky brown; females macropterous; distribution Florida; host Ceratiola ericoides ........ weemsi Henry (pale form)

- Dorsum pale whitish or yellowish brown, with numerous red spots ........... 10

10. Hemelytral membrane uniformly dark or fumate; males and females always macropterous; distribution Florida and South Carolina to Texas; hosts Asteraceae . . . psalliodes Reuter Hemelytral membrane pale translucent with two quadrate fumate blotches on outer margin; males macropterous, females often brachypterous with wing membrane reduced; distribution eastern Nebraska; host Asteraceae ............... cruentus, n.sp.

Parthenicus cruentus, new species figures $1,2,13-30$

Holotype 8 : USA: Nebraska: Johnson Co.: near Vesta, $40.35639^{\circ} \mathrm{N} 96.33751^{\circ} \mathrm{W}, 11$ Jul 1986, T.J. Henry and A.G. Wheeler, Jr. (AMNH_PBI 00070409) (USNM).

Diagnosis: This red-spotted species (figs. 1, 2), with macropterous males and macropterous and/or brachypterous females, is very similar to $P$. psalliodes Reuter in the overall pale color with small red spots over the dorsum, pale antennal segment II, and the infuscated hindfemora with small dark brown spots. It is distinguished by the overall smaller size, the translucent hemelytral membrane with two fumate or fuscous blotches on the margins, and the male genitalia (figs. 25-27), particularly the much more slender right paramere (fig. 26). In addition, $P$. cruentus has strongly brachypterous females within its populations, whereas I have never seen a brachypterous $P$. psalliodes among the numerous specimens examined from Florida to Texas.

DESCRIPTION: Macropterous male $(N=10$; holotype in parentheses): Length to apex of membrane $2.55-2.85 \mathrm{~mm}(2.60 \mathrm{~mm})$, length to base of cuneus $1.80-1.85 \mathrm{~mm}(1.75 \mathrm{~mm})$, width $1.06-1.12 \mathrm{~mm}(1.06 \mathrm{~mm})$. Head: Length $0.30-0.34 \mathrm{~mm}(0.32 \mathrm{~mm})$, width across eyes $0.53-0.56 \mathrm{~mm}(0.53 \mathrm{~mm})$, vertex between eyes $0.27-0.29 \mathrm{~mm} \quad(0.27 \mathrm{~mm})$. Labium: Length $1.06-1.09 \mathrm{~mm}(1.07 \mathrm{~mm})$, extending to third abdominal segment. Antenna: Segment I, length $0.24-0.27 \mathrm{~mm}(0.24 \mathrm{~mm})$; II, 0.88-0.91 $\mathrm{mm}(0.85 \mathrm{~mm})$; III, $0.56-0.62 \mathrm{~mm}(0.69 \mathrm{~mm})$; IV, $\quad 0.29-0.30 \mathrm{~mm} \quad(0.34 \mathrm{~mm})$. Pronotum: Length $0.38-0.40 \mathrm{~mm}$, basal width 0.83 $0.90 \mathrm{~mm}(0.80 \mathrm{~mm})$.

19 June 1989). 4, P. juniperi, female (same data as for male). 5, P. knighti, male (Florida, Fort Meyers, 12 May 1928). 6, P. knighti, female (same data as for male). 7, P. psalliodes, male (Texas, College Station, 9 May 1929). 8, P. psalliodes, female (Florida, Alachua, Gainesville, 28-29 April 1981). 9, P. rufus, male (South Carolina, Lexington Co., 0.4 km N of S65, $3.3 \mathrm{~km}$ E of Gaston, 5 June 1999). 10, P. rufus, female (Florida, Collier Co., $1.5 \mathrm{mi}$ E of Hendry Co. line, 25 April 1982). 11, P. sedumicola, male (Arkansas, Stone Co., Rt. 5, at Sylamore Creek Access, jct. Rts. 9 \& 14, 13 June 2001). 12, P. sedumicola, female (same data as for male). 

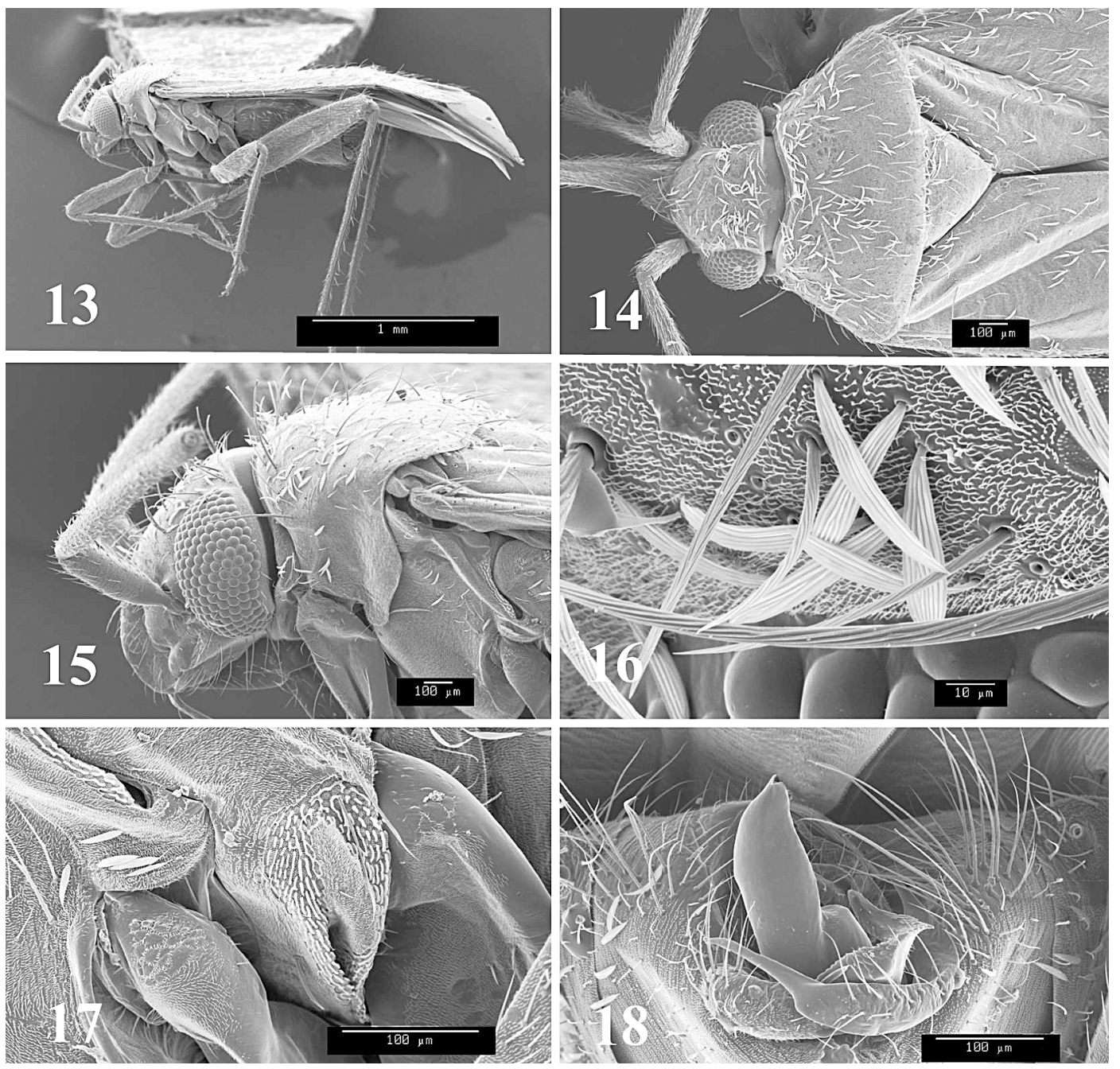

Figs. 13-18. Scanning electron photomicrographs of Parthenicus cruentus adult male. 13, Lateral aspect $(35.8 \times) .14$, Head and pronotum, dorsal aspect $(64.5 \times) .15$, Head and pronotum, lateral aspect $(121 \times) .16$, Scalelike setae on head $(1080 \times)$. 17, Ostiolar evaporative area $(304 \times)$. 18, Genital capsule, caudal aspect $(240 \times)$.

Macropterous female $(N=10)$ : Length to apex of membrane $2.30-2.85 \mathrm{~mm}$, length to base of cuneus $1.73-1.98 \mathrm{~mm}$, width 1.06 $1.12 \mathrm{~mm}$. Head: Length $0.34-0.37 \mathrm{~mm}$, width across eyes $0.54-0.56 \mathrm{~mm}$, vertex between eyes $0.29-0.32 \mathrm{~mm}$. Labium: Length 1.10 $1.12 \mathrm{~mm}$, extending to base of ovipositor. Antenna: Segment I, length 0.22-0.24 mm; II, 0.85-0.93 mm; III, $0.59-0.69 \mathrm{~mm}$; IV, $0.32-$ $0.37 \mathrm{~mm}$. Pronotum: Length $0.37-0.42 \mathrm{~mm}$, basal width $0.78-0.91 \mathrm{~mm}$.
Brachypterous female $(N=10)$ : Length to apex of membrane $2.08-2.14 \mathrm{~mm}$, length to base of cuneus $1.57-1.76 \mathrm{~mm}$, width $1.02-$ $1.04 \mathrm{~mm}$. Head: Length $0.29-0.32 \mathrm{~mm}$, width across eyes $0.54-0.56 \mathrm{~mm}$, vertex between eyes $0.30-0.32 \mathrm{~mm}$. Labium: Length 1.07$1.10 \mathrm{~mm}$, extending to base of ovipositor. Antenna: Segment I, length 0.21-0.22 mm; II, 0.78-0.84 mm; III, 0.61-0.66 mm; IV, 0.3$0.37 \mathrm{~mm}$. Pronotum: Length $0.32-0.34 \mathrm{~mm}$, basal width $0.74-0.77 \mathrm{~mm}$. COLORATION 

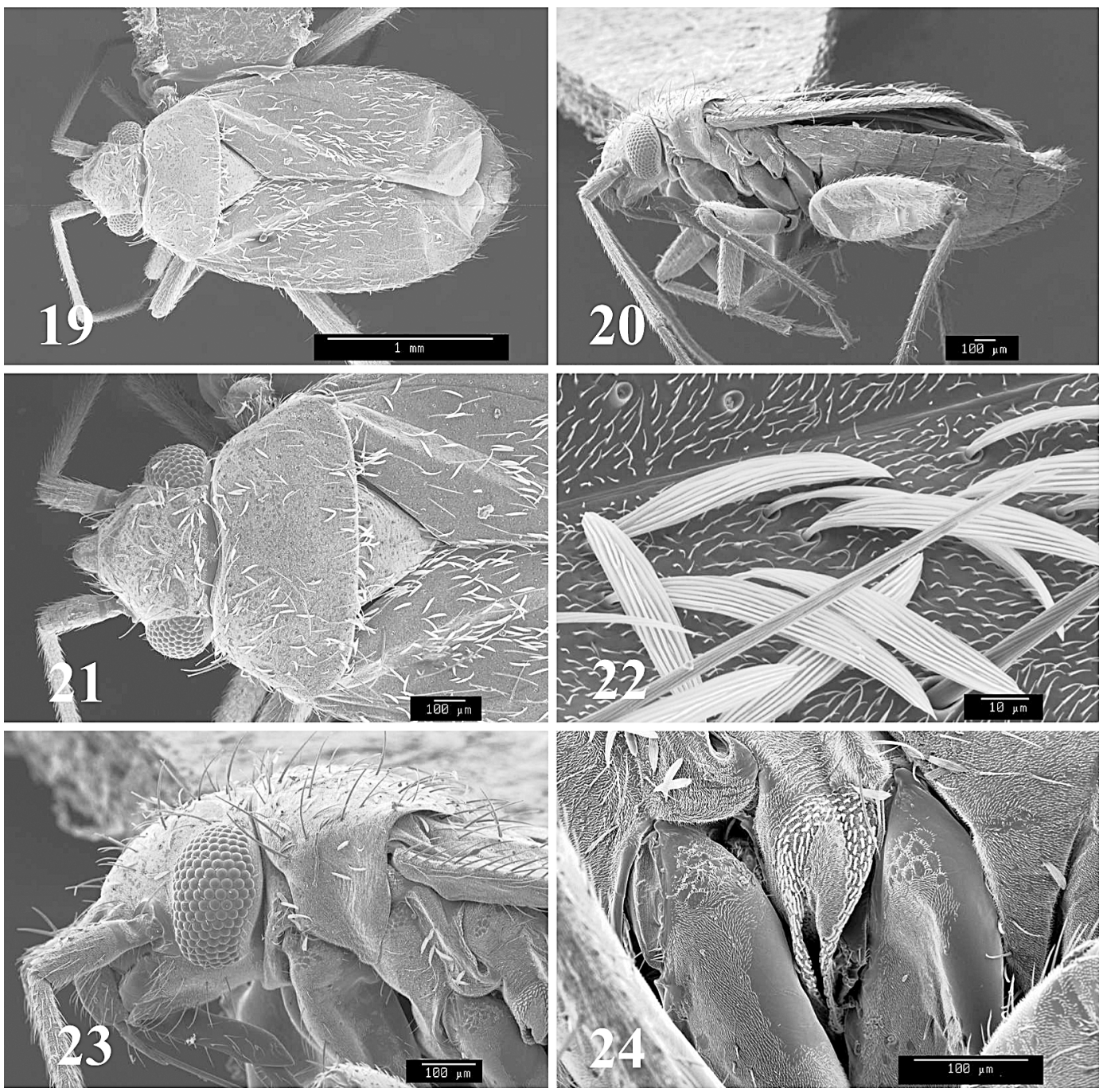

Figs. 19-24. Scanning electron photomicrographs of Parthenicus cruentus adult female. 19, Dorsal aspect $(37.2 \times)$. 20, Lateral aspect $(48.0 \times)$. 21, Head and pronotum, dorsal aspect $(71.5 \times)$. 22, Scalelike setae on head $(1080 \times)$. 23, Head and pronotum, lateral aspect $(121 \times)$. 24, Ostiolar evaporative area $(256 \times)$.

(figs. 1, 2): Overall coloration pale yellowish brown, densely intermixed with tiny red to brown spots. Head: Pale yellowish brown, with mottled brown markings on vertex and four to five indistinct transverse brown lines on either side of frons. Antenna: Segment I pale yellow to pale yellowish brown, sometimes with a narrow reddish- or brownish-red ring at base, particularly in females; II uniformly pale yellowish brown; III and IV yellowish brown, becoming dusky brown distally. Pronotum: Yellowish brown, shaded with darker brown across calli, densely interspersed with mostly small brown and some red (posteriorly) spots; mesoscutum brown; scutellum brown, becoming paler distally with a few small brown spots. Hemelytron: Pale yellowish brown, with numerous, evenly distributed, small, irregular red spots, with slightly fewer spots on cuneus; membrane clear or translucent brown, with two dark or fumate spots on margin (less distinct on brachypters), a smaller one near apex of cuneus and a slightly larger one just beyond, 

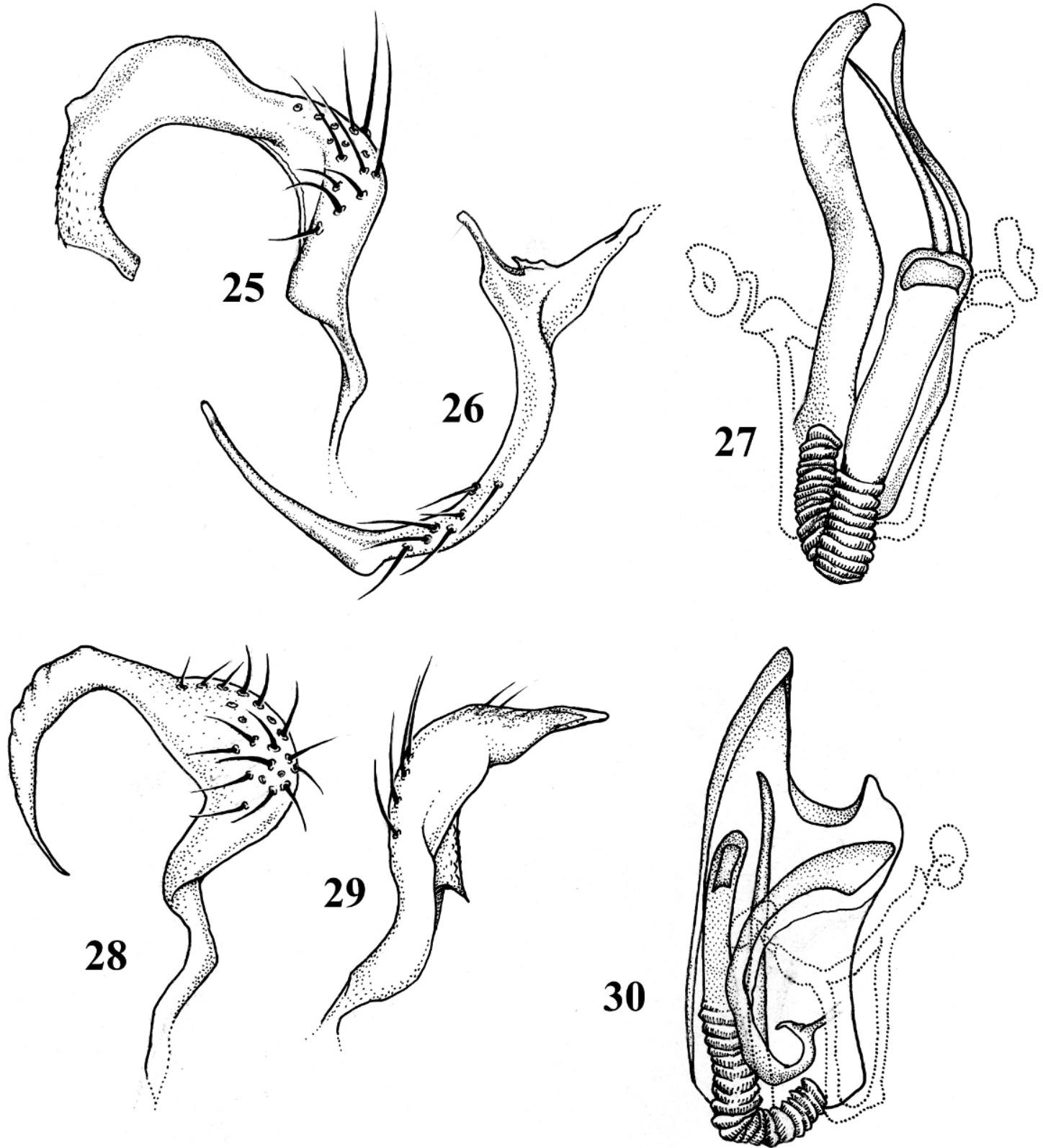

Figs. 25-27. Male genitalia of Parthenicus cruentus. 25, Left paramere. 26, Right paramere. 27, Vesica and phallotheca. Figs. 28-30. Male genitalia of Parthenicus psalliodes. 28, Left paramere. 29, Right paramere. 30, Vesica and phallotheca.

veins pale yellowish with small red spots on most areas. Ventral surface: Yellowish brown to brown, abdomen usually with a few red spots basally and often accented with dark brown. Legs: Femora yellowish brown to brown, densely speckled with dark brown spots on distal third to two-thirds, hindfemur becoming dark brown on some specimens but with brown spots still visible; tibiae pale yellowish brown with distinct red spots at bases of most pale brown spines, spots indistinct or absent on distal one-third of 
front and middle tibiae; tarsi pale yellowish brown; claws brown to fuscous. TEXTURE AND VESTITURE: Dorsum impunctate and semishiny. Head, pronotum, scutellum, and hemelytra with scattered long, erect and semierect, pale or whitish, simple setae, intermixed with white or silvery scalelike setae, the latter restricted on hemelytra mostly to clavus and basal half of corium; hemelytra also with black scalelike setae concentrated mostly on apical half of clavus and apical third of corium (and with a few on scutellum). Ventral surface with scattered simple setae and a few white scalelike setae on pleural areas of thorax and sides and ventral surface of abdomen. STRUCTURE: Males (fig. 1) fully macropterous with well-developed hemelytra. Females either fully macropterous or subbrachypterous (fig. 2) with the cuneus and membrane greatly shortened.

Male genitalia: Left paramere (fig. 25) strongly arching, stout at base, more slender distally; right paramere (fig. 26) slender, Cshaped; vesica (and phallotheca) (fig. 27) with one slender spiculum.

ETYMOLOGY: From the Latin "cruor", meaning spotted with red (blood), referring to the pale red-spotted dorsum of this species.

Hosts: Undetermined Asteraceae. The host and biology of this species are being investigated by A.G. Wheeler, Jr.

Distribution: Southeastern Nebraska.

PARATYPes: USA: Nebraska: Johnson Co.: near Vesta, $40.35639^{\circ} \mathrm{N} 96.33751^{\circ} \mathrm{W}, 11 \mathrm{Jul}$ 1986, T.J. Henry and A.G. Wheeler, Jr., $15 \hat{\jmath}$ (AMNH_PBI 00070443-00070456, AMNH_ PBI 00070459), 51 우 (AMNH_PBI 0007041000070442, AMNH_PBI 00070460, AMNH PBI 00070474-00070490) (AMNH, CNC, USNM).

\section{Parthenicus juniperi (Heidemann)}

figures 3,4

Psallus juniperi Heidemann, 1892: 225 (n.sp.), 1905: 49 (as n.sp.); Van Duzee, 1916: 46 (list), 1917: 406 (cat.). Lectotype designated by Wheeler and Henry, 1975: 361.

Apocremnus (Psallus) juniperi: Barber, 1914: 500 (list).

Parthenicus juniperi: Knight, 1919: 114 (comb., note), 1923: 499 (descrip., key); Blatchley, 1926: 806 (descrip., note, key); Knight, 1941: 76 (descrip., key, fig.); Froeschner, 1949: 166 (note, key); Carvalho, 1958: 123 (cat.); Akingbohungbe et al., 1972: 11 (list), 1973: 14 (descrip. nymph); Wheeler and Henry, 1975: 361 (note, type desig.), 1977: 637 (descrip., distr., hosts); Henry and Smith, 1979: 214 (list); Henry, 1982: 356 (descrip., distr., hosts, key, figs.); Wheeler et al., 1983: 142 (list, host); Snodgrass et al., 1984: 853 (list, host); Henry and Wheeler, 1988: 439 (cat.); Schuh, 1995: 179 (cat.); Maw et al., 2000: 119 (list); Henry et al., 2005: 61 (list, hosts).

Diagnosis: This species is distinguished from other eastern United States species by the testaceous dorsum (figs. 3, 4), often with a salmon-pink tinge, the infuscated scutellum, the dense black scalelike setae on the scutellum and apex of the corium, the contrastingly red cuneus, and the pale testaceous tibiae lacking spots at the bases of the spines. Both males and females of $P$. juniperi are always fully macropterous.

Hosts: Common on native eastern red cedar, Juniperus virginiana L., ornamental Chinese juniper, Juniperus chinensis L., and other species of Chamaecyparis, Juniperus, and Thuja (Cupressaceae) (Wheeler and Henry, 1977; Henry, 1982). Juniperus bermudensis L. is a new host record from Bermuda. All noncupressaceous hosts listed in the specimen data are accidental or merely sitting records.

DisTRIBUTION: This species, originally described from Washington, D.C., and Berkeley Springs, West Virginia (Heidemann 1892), later was reported from Canada (Ontario) and the following U.S. states: Florida, Georgia, Illinois, Indiana, Kentucky, Maryland, Massachusetts, Mississippi, Missouri, North Carolina, Nebraska, New York, North Carolina, Ohio, Pennsylvania, Virginia, and Wisconsin (Henry and Wheeler, 1988; Henry et al., 2005). Wheeler and Henry (1977) designated a lectotype from Washington, D.C. Henry (1982) indicated that this species is widespread east of the 100th Meridian. New U.S. state records are Alabama, Arkansas, Colorado, Connecticut, Iowa, Kansas, Michigan, Minnesota, New Jersey, Oklahoma, South Carolina, Tennessee, and Texas. Bermuda is a new record overlooked by Henry and Hillburn (1990).

TYPE MATERIAL ExAMINED: Lectotype $q$ : USA: District of Columbia: No specific locality, Washington, D.C., 19 Jun 1891, "Heide- 
mann Collection" (AMNH_PBI 00071228) (USNM).

Other SPeCimens ExAmined: BERMUDA: Smith's Parish Co.: Spittal Pond, $32.31194^{\circ} \mathrm{N}$ $64.72611^{\circ} \mathrm{W}, 1 \mathrm{~m}, 13$ Jan 1988, T.J. Henry, Juniperus bermudiana L. (Cupressaceae), 1 우 (AMNH_PBI 00070201)(USNM). CANADA: Ontario: Dunnville, $42.9^{\circ} \mathrm{N} 79.61666^{\circ} \mathrm{W}, 09 \mathrm{Jul}$ 1962, Kelton and Thorpe, 1 ㅇ (CNC). Effingham, $43.07^{\circ} \mathrm{N} 79.3^{\circ} \mathrm{W}, 12$ Jul 1955 , L.A. Kelton, 1하, 2 ㅇ (CNC); 11 Aug 1961, L. A. Kelton, $5 \hat{\delta}, 11$, $(\mathrm{CNC})$. Forest, $43.1^{\circ} \mathrm{N} 82^{\circ} \mathrm{W}$, 17 Jul 1962, Kelton and Brumpton, $J u$ niperus virginiana L. (Cupressaceae), 4ㅎ, 7 우 (CNC). Ipperwash Provincial Park, $43.21666^{\circ} \mathrm{N}$ $81.95416^{\circ} \mathrm{W}, 11 \mathrm{Jul} 1962$, Kelton and Thorpe, Juniperus virginiana L. (Cupressaceae), $13 \hat{\jmath}$, 10 우 (CNC). Jordan, $43.15^{\circ} \mathrm{N} 79.38^{\circ} \mathrm{W}, 17 \mathrm{Jul}$ 1961, L.A. Kelton, Juniperus virginiana L. (Cupressaceae), 15§̊, 9 우 (CNC); 9 Aug 1961, L.A. Kelton, Juniperus virginiana L. (Cupressaceae), 79 (CNC); 26 Sep 1961, L.A. Kelton, Juniperus virginiana L. (Cupressaceae), 12ઈิ, 26 ㅇ (CNC); 24 Aug 1961, Kelton and Brumpton, Juniperus virginiana L. (Cupressaceae), 1호, 5우 (CNC). Kingsville, $42.03333^{\circ} \mathrm{N} 82.75^{\circ} \mathrm{W}, 7$ Jul 1962, G. Thorpe, Juniperus virginiana L. (Cupressaceae), $3 \hat{\delta}$, 7 우 (CNC). Leamington, $42.05^{\circ} \mathrm{N} 82.58333^{\circ} \mathrm{W}$, 12 Sep 1961-13 Sep 1961, Kelton and Brumpton, Juniperus virginiana L. (Cupressaceae), 1 우 (CNC). Mount Pleasant, $44.23333^{\circ} \mathrm{N}$ 77.03333 ${ }^{\circ}$ W, 22 Aug 1961, L.A. Kelton, 1 우 (CNC). Nepean, Piney Forest, Lafontaine House, $45.31754^{\circ} \mathrm{N} 75.73024^{\circ} \mathrm{W}, 102 \mathrm{~m}, 18 \mathrm{Jul}$ 1991, M.D. Schwartz, 1 ㅇ (CNC). Niagara Falls, $43.25^{\circ} \mathrm{N} 79.06666^{\circ} \mathrm{W}, 14$ Aug 1961, L.A. Kelton, Fraxinus sp. (Oleaceae), 1 ㅇ (CNC). Normandale, $42.72^{\circ} \mathrm{N} 80.32^{\circ} \mathrm{W}, 13$ Jul 1962 , H. Blanchard, Juniperus virginiana L. (Cupressaceae), $3 \hat{\delta}, 3 \hat{q}$ (CNC). Point Pelee, $41.96666^{\circ} \mathrm{N} 82.51666^{\circ} \mathrm{W}, 13$ Sep 1961, G. Brumpton, Juniperus virginiana L. (Cupressaceae), $1 \hat{\delta}, 1+$ (CNC). Turkey Point, $42.7^{\circ} \mathrm{N}$ $80.32^{\circ} \mathrm{W}, 4$ Jul 1962, G. Thorpe, Pinus banksiana Lamb. (Pinaceae), $1 \hat{\delta}$ (CNC). Vineland, $43.15^{\circ} \mathrm{N} 79.4^{\circ} \mathrm{W}, 1$ Aug $1977-5$ Aug 1977, L.A. Kelton, Juniperus virginiana L. (Cupressaceae), $10 \hat{\jmath}, 19$ Catalpa sp. (Bignoniaceae), 1ㅎ, $3 ㅇ$ (CNC). USA: Alabama: Washington Co.: LeRoy, $31.50461^{\circ} \mathrm{N} 87.98472^{\circ} \mathrm{W}, 12$ Jun 1917 , H.H. Knight, 1 ช (AMNH_PBI 00070202), 1 우
(AMNH_PBI 00070203) (USNM). Arkansas: Drew Co.: Monticello University, $33.59039^{\circ} \mathrm{N}$ 91.81319 ${ }^{\circ}$ W, 9 Jun 1987, H.H. Knight, Juniperus virginiana L. (Cupressaceae), 1 우 (AMNH_PBI 00070204) (USNM). Faulkner Co.: Conway, Hendrix College, $35.09925^{\circ} \mathrm{N} 92.44182^{\circ} \mathrm{W}, 12$ Jun 1987, T.J. Henry and A.G. Wheeler, Thuja occidentalis L. (Cupressaceae), $1 \hat{\sigma}$ (AMNH_ PBI 00070205) (USNM). Garland Co.: Rt. 298, $5.1 \mathrm{mi}$. W. of Rt. $7,34.70483^{\circ} \mathrm{N} 93.14733^{\circ} \mathrm{W}, 08$ Jun 2004, T.J. Henry and A.G. Wheeler, Jr, Juniperus virginiana L. (Cupressaceae), 2 ㅇ (AMNH_PBI 00070206-00070207) (USNM). Johnson Co.: Clarksville, University of Ozarks, $35.47533^{\circ} \mathrm{N} 93.46783^{\circ} \mathrm{W}, 9$ Jun 2004 , T.J. Henry and A.G. Wheeler, Jr., Juniperus chinensis L. (Cupressaceae), $1 \delta^{\hat{\sigma}}$ (AMNH_PBI 00070208), 79 (AMNH_PBI 00070209-00070215)(USNM). Newton Co.: Rt. 16, overlook at Deer, $35.82033^{\circ} \mathrm{N} 93.21633^{\circ} \mathrm{W}, 11$ Jun 2004, T.J. Henry and A.G. Wheeler, Jr., Juniperus virginiana L. (Cupressaceae), $3 \hat{\delta}$ (AMNH_PBI 0007021800070220), 2 q (AMNH_PBI 00070216-00070217) (USNM). Pope Co.: Russellville, Arkansas Tech. University, $35.27842^{\circ} \mathrm{N} 93.13379^{\circ} \mathrm{W}, 13$ Jan 1987 , T.J. Henry and A.G. Wheeler, Jr., Juniperus virginiana L. (Cupressaceae), 1 우 (AMNH_PBI 00070221) (USNM). Washington Co.: Durham on White River (Middle Fork), $35.94952^{\circ} \mathrm{N}$ $93.98048^{\circ} \mathrm{W}, 382 \mathrm{~m}, 27$ Jul 1986, M.D. Schwartz, Juniperus virginiana L. (Cupressaceae), $2 \hat{\delta}$ (AMNH_PBI 00071309-00071310), 15 \% (AMNH_PBI 0007131-00071325) (AMNH). Juniperus virginiana L. (Cupressaceae), 20, 1 우 (CNC). Fayetteville, University of Arkansas campus, $36.06258^{\circ} \mathrm{N} 94.15743^{\circ} \mathrm{W}, 15$ Jun 1987 , T.J. Henry and A.G. Wheeler, Jr., Juniperus virginiana L. (Cupressaceae), $2+$ (AMNH_PBI 0007022200070223) (USNM). Colorado: Larimer Co.: Fort Collins, Colorado State University Campus, $40.57^{\circ} \mathrm{N} 105.08^{\circ} \mathrm{W}, 18$ Aug 2005, T.J. Henry and A.G. Wheeler, Jr., Juniperus chinensis L. (Cupressaceae), $6 \hat{\delta}$ (AMNH_PBI 0007123000071235), 1 오요. Connecticut: Middlesex Co.: Wesleyan University, Middletown, $41.55565^{\circ} \mathrm{N} 72.65565^{\circ} \mathrm{W}, 15 \mathrm{Jul} 1989$, A.G. Wheeler, Jr., Juniperus chinensis L. (Cupressaceae), $3+$ (AMNH_PBI 00070224-00070226) (USNM). District of Columbia: Washington, D.C., $38.90817^{\circ} \mathrm{N} 77.05105^{\circ} \mathrm{W}, 19$ Jul 1926, H.H. Knight, 10, 1 우 (CNC). Washington D.C., $38.89178^{\circ} \mathrm{N} 77.00831^{\circ} \mathrm{W}, 30$ Jun $1926-19$ Jul 1926, 
H.H. Knight, $1 \delta$ (AMNH_PBI 00070227), 31 (AMNH_PBI 00070228-00070258) (USNM); 12 Jul 1891, Unknown, 1 ô (AMNH_PBI 00070265) (USNM); 19 Jun 1891, Unknown, 1 을 $\left(\mathrm{AMNH}_{-}\right.$ PBI 00070266) (USNM). Florida: Brevard Co.: Merritt Island National Wildlife Refuge, near draw bridge, $28.73833^{\circ} \mathrm{N} 80.75116^{\circ} \mathrm{W}, 12$ May 2003 , T.J. Henry and A.G. Wheeler, Jr., Juniperus virginiana L. (Cupressaceae), 1 i (AMNH_PBI 00070259) (USNM). Highlands Co.: Rt 70, $3 \mathrm{mi} \mathrm{W}$ of Rt 27, near Archbold Biological Station, $27.2^{\circ} \mathrm{N} 81.38^{\circ} \mathrm{W}$, 30 Apr 1982, T.J. Henry and A.G. Wheeler, Jr., 1 t (AMNH_PBI 00070260) (USNM). Volusia Co.: South Daytona, $29.16556^{\circ} \mathrm{N} 81.00472^{\circ} \mathrm{W}, 19 \mathrm{Apr}$ 1959, J.F. Brimley, 1 우 (CNC). Georgia: DeKalb Co.: Stone Mountain, $33.80816^{\circ} \mathrm{N} 84.1702^{\circ} \mathrm{W}$, 8 Jun 1917, H.H. Knight, 1 우 (AMNH_PBI 00070261) (USNM). Peach Co.: Peach County, $32.5666^{\circ} \mathrm{N} 83.8333^{\circ} \mathrm{W}, 12$ Jul 1943 , Turner, Light Trap, 19 (AMNH_PBI 00070262) (USNM). Indiana: Putnam Co.: $39.6444^{\circ} \mathrm{N} 86.8647^{\circ} \mathrm{W}, 1$ Oct 1923, W.S. Blatchley, 1 우 (AMNH_PBI 00070263) (USNM). Iowa: Linn Co.: PalisadesKepler State Park, $41.90751^{\circ} \mathrm{N} 91.50629^{\circ} \mathrm{W}, 8 \mathrm{Jul}$ 1950, Laffoon, Slater, Hicks, $1+$ (AMNH_PBI 00070350) (USNM). Monroe Co.: Albia, $41.02667^{\circ} \mathrm{N} 92.80556^{\circ} \mathrm{W}, 15 \mathrm{Jul} 1927$, Harris and Johnston, cedar, $1 \hat{\delta}$ (CNC). Kansas: Crawford Co.: Pittsburg State University, Pittsburg, $37.41088^{\circ} \mathrm{N}$ $94.70496^{\circ} \mathrm{W}$, 7 Jul 2000, A.G. Wheeler, Jr., Juniperus virginiana L. (Cupressaceae), $1 \hat{\delta}$ (AMNH_PBI 00070351), 19 (AMNH_PBI 00070352) (USNM). Riley Co.: Manhattan, Kansas State University, $39.1965^{\circ} \mathrm{N} 96.57616^{\circ} \mathrm{W}$, 375 m, 22 Jun 1999, T.J. Henry and A.G. Wheeler, Jr., Juniperus virginiana L. (Cupressaceae), $1 \hat{\delta}$ (AMNH_PBI 00070353), $2{ }^{\circ}$ (AMNH_PBI 00070354, AMNH_PBI 00070355) (USNM).

Kentucky: Bullitt Co.: Bernheim Arboretum and Research Forest, $37.91833^{\circ} \mathrm{N} 85.66716^{\circ} \mathrm{W}$, 22 Jun 2001, T.J. Henry and A.G. Wheeler, Jr., Juniperus chinensis L. (Cupressaceae), $1 \hat{\delta}$ (AMNH_PBI 00070356) (USNM). Fayette Co.: Lexington, University of Kentucky Campus, $38.03483^{\circ} \mathrm{N}$ $84.5025^{\circ} \mathrm{W}, 17$ Jun 2001, T.J. Henry and A.G. Wheeler, Jr., Juniperus chinensis L. (Cupressaceae), 2 ㅇ (AMNH_PBI00070357-00070358) (USNM). Franklin Co.: Frankfort, $38.20091^{\circ} \mathrm{N} 84.87328^{\circ} \mathrm{W}$, 7 Jun 1991, A.G. Wheeler, Jr., Juniperus virginiana L. (Cupressaceae), 1 우 (AMNH_PBI 00070359) (USNM). Simpson Co.: Rt. 591 \& 383 at Providence, 4 Jun 1985, T.J. Henry and A.G.
Wheeler, Jr., Juniperus virginiana (Cupressaceae), 20ิ (AMNH_PBI 00070360-00070361) (USNM). Maryland: Montgomery Co.: Cabin John Bridge, $38.97528^{\circ} \mathrm{N} 77.15833^{\circ} \mathrm{W}, 29$ Jul 1914, H.S. Barber, 1 ( (AMNH_PBI 00070272) (USNM). Plummers Island, $38.97049^{\circ} \mathrm{N} 77.1763^{\circ} \mathrm{W}, 8$ Jun 1913, W.L. McAtee, 5ㅇ (AMNH_PBI 00070368-00070371, AMNH_PBI 00070374) (USNM); 13 Sep 1914, W.L. McAtee, 5ㅇ (AMNH_PBI 0007036200070366) (USNM); 5 Jul 1914, W.L. McAtee, 1 우 (AMNH_PBI 00070367) (USNM); 4 Sep 1913, W.L. McAtee, 1 우 (AMNH_PBI 00070373) (USNM); 12 Oct 1913, W.L. McAtee, 1 우 (AMNH_PBI 00070372) (USNM). Rockville, $39.084^{\circ} \mathrm{N} 77.15276^{\circ} \mathrm{W}, 19$ Jun 1989 , W.E. Steiner and J.M. Swearingen, $3 \hat{\delta}$ (AMNH_PBI0007027300070275) (USNM). St. Mary's Co.: Calloway [Landing], 38.16874 ${ }^{\circ} \mathrm{N} 76.46079^{\circ} \mathrm{W}, 26$ Aug 1946, R.I. Sailer, $2+$ (AMNH_PBI 00070276-00070277) (USNM). Massachusetts: Dukes Co.: Marthas Vineyard, $41.33333^{\circ} \mathrm{N} 70.61611^{\circ} \mathrm{W}, 24 \mathrm{~m}, 13 \mathrm{Aug}$

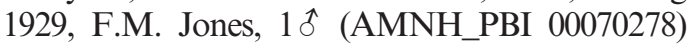
(USNM). Michigan: Ingham Co.: Dansville, Fairview Cemetary, $42.55587^{\circ} \mathrm{N} 84.3033^{\circ} \mathrm{W}, 15$ Jul 1991, T.J. Henry and A.G. Wheeler, Jr., Juniperus virginiana L. (Cupressaceae), 3 우 (AMNH_PBI 00070279-00070281) (USNM). Minnesota: Ramsey Co.: Saint Anthony Park, $44.97805^{\circ} \mathrm{N} 93.19083^{\circ} \mathrm{W}, 25$ Jul 1924, H.H. Knight, 20 (AMNH_PBI 00070284-00070285) (USNM). Washington Co.: Grey Cloud Island, $44.79916^{\circ}$ N $93.00666^{\circ}$ W, 20 Jul 1920, H.H. Knight, Juniperus virginiana L. (Cupressaceae), 2 ㅇ (AMNH_PBI 00070282-00070283) (USNM). Missouri: Ozark Co.: Gainesville, Rt.181 at Jct. $160,36.60816^{\circ} \mathrm{N} 92.4155^{\circ} \mathrm{W}, 14$ Jun 2004 , T.J. Henry and A.G. Wheeler, Jr., Juniperus virginiana L. (Cupressaceae), $2+$ (AMNH_PBI 0007028600070287) (USNM). Nebraska: Adams Co.: Hastings, Hastings College Campus, $40.59168^{\circ} \mathrm{N}$ $98.37311^{\circ} \mathrm{W}, 12$ Jul 1986, T.J. Henry and A.G. Wheeler, Jr., Juniperus sp. (Cupressaceae), $2 \hat{\jmath}$ (AMNH_PBI 00070288-00070289), 2 ㅇ (AMNH_ PBI 00070290-00070291) (USNM). Antelope Co.: Royal, $42.33222^{\circ} \mathrm{N} 98.12556^{\circ} \mathrm{W}, 27$ Jul 1973, L.A. Kelton, Juniperus virginiana L. (Cupressaceae), 7 $\delta$, $6+$ (CNC). Brown Co.: Long Pine, $42.53639^{\circ} \mathrm{N}$ 99.70028 W, 27 Jun 1973, L.A. Kelton, Juniperus

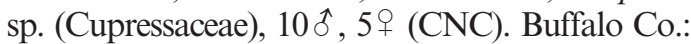
Odessa, $40.70168^{\circ} \mathrm{N} 99.25676^{\circ} \mathrm{W}, 677 \mathrm{~m}, 7$ Sep

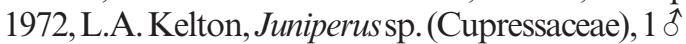
(CNC). Fillmore Co.: Fairmont, Jct. 6 \& 81, 
$40.63695^{\circ} \mathrm{N} 97.58533^{\circ} \mathrm{W}, 12 \mathrm{Jul} 1986$, T.J. Henry and A.G. Wheeler, Jr., Juniperus scopulorum L. (Cupressaceae), 19 (AMNH_PBI 00070292) (USNM). Garden Co.: Wood Lake, $41.91664^{\circ} \mathrm{N}$ $102.3501^{\circ}$ W, 27 Jun 1973, L.A. Kelton, Juniperus

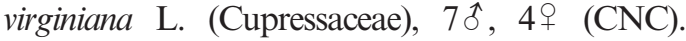
Nemaha Co.: Auburn, at cemetery, $40.39278^{\circ} \mathrm{N}$ $95.83889^{\circ} \mathrm{W}, 11$ Jul 1986, T.J. Henry and A.G. Wheeler, Jr., Juniperus sp. (Cupressaceae), 1 i (AMNH_PBI 00070295) (USNM). Peru, Neal Park, $40.46972^{\circ} \mathrm{N} 95.73277^{\circ} \mathrm{W}, 9$ Jul 1986, T.J. Henry and A.G. Wheeler, Jr., Juniperus chinensis L. (Cupressaceae), 1 ㅇ (AMNH_PBI 00070294) (USNM). Rt. 75, near Auburn \& Little Nemaha River, 10 Jul 1986, T.J. Henry and A.G. Wheeler, Jr., Juniperus sp. L. (Cupressaceae), $1 \hat{\jmath}$ (AMNH_ PBI 00070293) (USNM). New Jersey: Ocean Co.: Mystic Island, Radio Road on Bay, $39.5394^{\circ} \mathrm{N}$ 74.3883 ${ }^{\circ} \mathrm{W}, 1$ m, 4 Jul 1977, T.J. Henry, Juniperus virginiana L. (Cupressaceae), $1 \hat{\text { }}$ (AMNH_PBI 00070270) (USNM). Seaside Heights Park, $39.92667^{\circ} \mathrm{N} 74.0775^{\circ} \mathrm{W}, 3$ Jul 1977, T.J. Henry, Juniperus sp. L. (Cupressaceae), $2 \hat{\jmath}$ (AMNH_PBI 00070264, AMNH_PBI 00070267), 2 ㅇ (AMNH_ PBI 00070268-00070269) (USNM). New York: Nassau Co.: Muttontown Park and Preserve, East Norwich, $40.84667^{\circ} \mathrm{N} 73.53556^{\circ} \mathrm{W}, 14 \mathrm{Jul} 1985$, M.D. Schwartz, Juniperus virginiana L. (Cupressaceae), 15 오 (AMNH_PBI 00071241-00071254, AMNH_PBI 00071308) (AMNH). Old Westbury, Bacon Road, $40.778^{\circ} \mathrm{N} 73.6185^{\circ} \mathrm{W}, 43 \mathrm{~m}$, 7 Aug 1988, M.D. Schwartz, Juniperus virginiana L. (Cupressaceae), 3 ㅇ (AMNH_PBI 0007132900071331) (AMNH). Roslyn, Cemetery on Rt 25A, 40.80902 ${ }^{\circ} \mathrm{N} 73.63199^{\circ} \mathrm{W}, 60 \mathrm{~m}, 30$ Jun 1985 , M.D. Schwartz, 9우 (AMNH_PBI 0007129800071306) Thuja occidentalis L. (Cupressaceae), $6{ }^{\hat{}}$ (AMNH_PBI 00070375-00070380) Thuja occidentalis L. (Cupressaceae), $1 \hat{\text { }}$ (AMNH_PBI 00071240), 5 ㅇ (AMNH_PBI 00070382, AMNH_ PBI 00071236-00071239) Tsuga canadensis L. (Cupressaceae), 1 우 (AMNH_PBI 00070381) (AMNH). Tsuga canadensis (L.) Carr. (Pinaceae), $1 \hat{\delta}$ Thuja occidentalis L. (Cupressaceae), 2̂े (CNC). Queens Co.: Flushing Meadow, Corona Park near zoo, $40.74326^{\circ} \mathrm{N} 73.86053^{\circ} \mathrm{W}, 8 \mathrm{~m}, 13$ Jul 1985, M.D. Schwartz, 1 우 (AMNH_PBI 00071307) (AMNH). Rockland Co.: Rockland Lake State Park, $41.13833^{\circ} \mathrm{N} 73.9225^{\circ} \mathrm{W}, 26$ Jun 1988, M.D. Schwartz, Juniperus virginiana L. (Cupressaceae), $3 \hat{\jmath}$ (AMNH_PBI 0007132600071328), 1 우 (AMNH_PBI 00071332)
(AMNH). Juniperus virginiana L. (Cupressaceae), $1 \hat{\text { o }}$ (CNC). West Nyack, NY, $41.09649^{\circ} \mathrm{N} 73.97292^{\circ} \mathrm{W}, 22$ Sep 1921, C.E. Olsen, 1 ô (AMNH_PBI 00070271)(USNM). Suffolk Co.: Cold Spring Harbor, Long Island, $40.87678^{\circ} \mathrm{N} 73.47043^{\circ} \mathrm{W}, 26$ Jul 1920 , H.M. Parshley, 1 iq (AMNH_PBI 00070327) (USNM); 25 Jul 1902, H.G. Barber, Juniperus virginiana $\mathrm{L}$. (Cupressaceae), $1 \hat{\delta}$ (AMNH_PBI 00070328), 8 ㅇ (AMNH_PBI 00070329-00070336) (USNM). Coram, Penniquid Barrens, $40.86861^{\circ} \mathrm{N}$ $73.50138^{\circ} \mathrm{W}, 29 \mathrm{~m}$, Engelhardt, $1 \hat{\jmath}$ (AMNH_PBI 00070326)(USNM). East Quogue, Quogue Wildlife Refuge, $40.84056^{\circ} \mathrm{N} 72.58194^{\circ} \mathrm{W}, 19 \mathrm{Jul} 1988$, M.D. Schwartz, Juniperus virginiana L. (Cupressaceae), 1 of (CNC). Riverhead, $40.91704^{\circ} \mathrm{N} 72.66204^{\circ} \mathrm{W}$, 10 May 1953, R. Latham, $1 \hat{\delta}$ (AMNH_PBI 00070337) (USNM). Tompkins Co.: Taghanic [sic, Taughannock], Ithaca, $42.5454^{\circ} \mathrm{N} 76.604^{\circ} \mathrm{W}$, 152 m, 11 Jul 1920, H.H. Knight, Juniperus virgin-

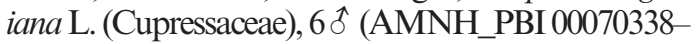
00070343), 1 오 (AMNH_PBI 00070344) (USNM). North Carolina: Mecklenburg Co.: near Matthews, Rt $511 \mathrm{mi} \mathrm{W}$ of Rt $16,35.11667^{\circ} \mathrm{N}$ $80.72389^{\circ}$ W, 2 Sep 1973, A.G. Wheeler, Jr., Juniperus virginiana L. (Cupressaceae), $1 \hat{\delta}$ (AMNH_PBI 00070345) (USNM). Oklahoma: Alfalfa Co.: Unknown locality, $36.72071^{\circ} \mathrm{N}$ 98.33717 W, 21 Jul 1949-10 Sep 1949, S. Coppock, Jr., 20 (AMNH_PBI 0007034600070347), 1 ( (AMNH_PBI 00070348) (USNM). Cherokee Co.: Northeastern State University, Tahlequah, 18 June $1999,35.91537^{\circ} \mathrm{N} 94.96996^{\circ} \mathrm{W}$, 18 Jun 1999, T.J. Henry and A.G. Wheeler, Jr., Juniperus virginiana L. (Cupressaceae), 1 ㅇ (AMNH_PBI 00070349) (USNM).Cleveland Co.: Norman University, $35.20266^{\circ} \mathrm{N} 97.44233^{\circ} \mathrm{W}$, 300 m, 13 Jun 1999, T.J. Henry and A.G. Wheeler, Jr., Juniperus chinensis L. (Cupressaceae), 1 ㅇ (AMNH_PBI 00070309) (USNM). Pennsylvania: Dauphin Co.: Harrisburg Cemetery, $40.26981^{\circ} \mathrm{N}$ 76.87358 ${ }^{\circ} \mathrm{W}, 22$ Aug 1972, collector unknown, Juniperus virginiana L.(Cupressaceae), $1 \delta$ (AMNH_ PBI 00070310) (USNM). Franklin Co.: Chambersburg, $39.9375^{\circ} \mathrm{N} 77.66139^{\circ} \mathrm{W}, 8$ Aug 1922 , J.R.

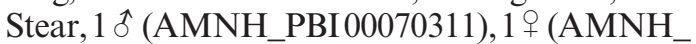
PBI 00070312) (USNM). Monroe Co.: Sciota Flea Market, Drive In Movie, $40.9345^{\circ} \mathrm{N}$ $75.3201^{\circ}$ W, 183 m, 7 Jul 1985, M.D. Schwartz,

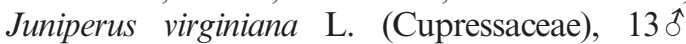
(AMNH_PBI 00071255-00071267), 30ㅇ (AMNH PBI 00071268-00071297) (AMNH). Juniperus vir- 
giniana L. (Cupressaceae), $2 \hat{\delta}$ (CNC). South Carolina: Lancaster Co.: 40 Acre Rock near Taxanaw, 7 May 1989, A.G. Wheeler, Jr., Juniperus virginiana $\mathrm{L}$. (Cupressaceae), $1 \hat{\delta}$ (AMNH_PBI 00070313) (USNM). Oconee Co.: Seneca, $34.68556^{\circ} \mathrm{N} 82.95333^{\circ} \mathrm{W}, 20$ Aug 1957, W.R. Richards, $1+$ (CNC). Orangeburg Co.: Edisto Gardens, $33.47639^{\circ} \mathrm{N} 80.89889^{\circ} \mathrm{W}, 06$ May 1989 , A.G. Wheeler, Jr., Juniperus virginiana L. (Cupressaceae), $1 \hat{\sigma}$ (AMNH_PBI 00070315) (USNM). York Co.: Winthrop College, Rock Hill, $34.9407^{\circ} \mathrm{N} 81.02841^{\circ} \mathrm{W}, 2$ Jul 1988, A.G. Wheeler, Jr., Juniperus virginiana L. (Cupressaceae), $1 \hat{\jmath}$ (AMNH_PBI 00070314) (USNM). Tennessee: Cannon Co.: Woodbury, $35.82757^{\circ} \mathrm{N} 86.07166^{\circ} \mathrm{W}$, 28 May 1985, T.J. Henry and A.G. Wheeler, Jr., Juniperus virginiana L. (Cupressaceae), 3 ㅇ (AMNH_ PBI 00070316-00070318)(USNM). Rutherford Co.: Middle Tennessee State University campus, Murfreesboro, $35.84556^{\circ} \mathrm{N} 86.39028^{\circ} \mathrm{W}, 28$ May 1985 , T.J.Henry and A.G. Wheeler, Jr., Juniperus virginiana L. (Cupressaceae), 29 (AMNH_PBI 0007031900070320) (USNM). Texas: Bastrop Co.: Pope Bend Road, $1 \mathrm{mi} \mathrm{N}$ of Rt 71, $3.7 \mathrm{mi} \mathrm{E}$ of Travis Co. line, $30.15527^{\circ} \mathrm{N} 97.45083^{\circ} \mathrm{W}, 168 \mathrm{~m}, 10$ May 2002, T.J. Henry and P.S.F. Ferreira, Juniperus virginiana L. (Cupressaceae), $1 \hat{\text { }}$ (AMNH_PBI 00070321), 1 오 (AMNH_PBI 00070322) (USNM). Brazos Co.: College Station, $30.62778^{\circ} \mathrm{N}$ 96.33417 W, 7 Nov 1981, T.J. Henry, Juniperus chinensis L. (Cupressaceae), $1 \hat{\delta}$ (AMNH_PBI 00070325), 2 ㅇ (AMNH_PBI 00070323-00070324) (USNM). Lee Co.: Rt. 21, West Yegua Creek at Lincoln, $30.28916^{\circ} \mathrm{N} 96.96066^{\circ} \mathrm{W}, 10$ May 2002, T.J. Henry and A.G. Wheeler, Jr., Juniperus virginiana L. (Cupressaceae), 29 (AMNH_PBI 0007029600070297) (USNM). Victoria Co.: Victoria, $28.805^{\circ} \mathrm{N} 97.00333^{\circ} \mathrm{W}, 24 \mathrm{Apr} 1983$, T.J. Henry and A.G. Wheeler, Jr., Juniperus chinensis hetzi (Cupressaceae), 1 ( (AMNH_PBI 00070298) (USNM). Virginia: Arlington Co.: Unknown locality, 22 Aug 1943, R.I. Sailer, $2 \hat{\delta}$ (AMNH_PBI 0007029900070300), 3 ㅇ (AMNH_PBI 00070301-00070303) (USNM). Essex Co.: 1 mi. SE of Dunnville, $37.86666^{\circ} \mathrm{N} 76.8^{\circ} \mathrm{W}, 27$ Jul 1993, T.J. Henry, Juniperus virginiana L. (Cupressaceae), $3 \circ$ (AMNH_ PBI 00070306-00070308) (USNM). Fairfax Co.: Herndon, $38.96983^{\circ} \mathrm{N} 77.3861^{\circ} \mathrm{W}, 1$ Aug 1911, H.G. Barber, Juniperus sp. (Cupressaceae), 1 + (AMNH_ PBI 00070304) (USNM). Wythe Co.: Wytheville, $36.94262^{\circ} \mathrm{N} 81.04564^{\circ} \mathrm{W}, 11$ Jul 1954, R.L. Hoffman, $1+$ (AMNH_PBI 00070305) (USNM).

\author{
Parthenicus knighti Henry \\ figures 5,6
}

Parthenicus knighti Henry, 1982: 359 (n.sp.); Henry and Wheeler, 1988: 439 (cat.); Schuh, 1995: 179 (cat.).

Diagnosis: This species, known only from macropterous specimens, is distinguished by the pale legs and antennal segments I and II; testaceous dorsum (figs. 5, 6), accented with red at the base and outside margin of the clavus, the inside margin of the corium, and the basal area of the cuneus; and by the long rostrum that extends to the fifth abdominal segment in males and to the base of the ovipositor in the only known female.

Host: Unknown. A handwritten label on one paratype male from Ft. Meyers indicates that it was collected (May 12) on a tidal flat, suggesting that the host is a coastal inhabitant, perhaps a halophytic plant.

Distribution: Known only from coastal areas of Hillsborough (Tampa) and Lee (Ft. Meyers) Counties, Florida (Henry 1982).

Type Material Examined: Holotype $\delta$ : USA: Florida: Lee Co.: Fort Myers, $26.64028^{\circ} \mathrm{N} 81.8725^{\circ} \mathrm{W}, 12$ May 1928, E.D. Ball (AMNH_PBI 00070498) (USNM). PARATYPES: USA: Florida: Hillsborough Co.: Tampa, $27.94722^{\circ} \mathrm{N} 82.45861^{\circ} \mathrm{W}, 10$ Sep 1927, E.D. Ball, 1 o (AMNH_PBI 00070497) (USNM). Lee Co.: Fort Myers, $26.64028^{\circ} \mathrm{N} 81.8725^{\circ} \mathrm{W}$, 12 May 1928, E.D. Ball $2 \hat{\sigma} \quad\left(\mathrm{AMNH}_{-}\right.$ PBI 00070495, AMNH_PBI 00070496), $4 \dot{+}$ (AMNH_PBI 00070491-00070494) (USNM).

Other SPeCimens ExAmined: USA: Florida: Pinellas Co.: Pine Key, $27.6918^{\circ} \mathrm{N} 82.7187^{\circ} \mathrm{W}$, 1 m, 24 Apr 1961-26 Apr 1961, L.A. Kelton, $17 \hat{\jmath}, 6$ 우 (CNC).

\section{Parthenicus psalliodes Reuter figures $7,8,28-30$}

Parthenicus psalliodes Reuter, 1876: 85 (n.sp.); Uhler, 1886: 20 (list); Atkinson, 1890: 135 (cat.); Van Duzee, 1916: 45 (list), 1917: 401 (cat.), 1918: 302 (key); Blatchley, 1926: 805 (descrip., note, key); Carvalho, 1952: 74 (as type), 1958: 123 (cat.); Henry, 1982: 361 (descrip., distr., hosts, key, figs.); Henry and Wheeler, 1988: 440 (cat.); Schuh, 1995: 180 (cat.). 
Diagnosis: This species, known only from macropters, is very similar to $P$. cruentus in the overall pale background color, interspersed with numerous small red spots. It is distinguished by the generally larger size, uniformly dark or fumate hemelytral membrane, and male genitalia (figs. 28-30). The left paramere (fig. 28) also is similar to that of $P$. cruentus, but it is more evenly rounded on the arching $\mathrm{C}$; the right paramere (fig. 29) is straighter, more stout, and has a slender apical process and lateral comb; and the vesica (fig. 30) possesses one slender and one broad, apically bifurcate spiculum.

Hosts: Woody goldenrod, Chrysoma pauciflosculosa (Michx.) Greene (Asteraceae); yankeeweed, Eupatorium compositifolium Walt (Asteraceae); Eupatorium $\times$ pinnatifidum Ell. (capillifolium $\times$ perfoliatum); and Texas snakeweed, Gutierrezia texana (DC.) Torreya and Gray (Asteraceae) (Henry, 1982). One specimen examined is labeled as feeding on foliage of desertbroom, Baccharis sarothroides Gray (Asteraceae).

Distribution: This species was described from Texas (Reuter, 1876) and later recorded from Alabama, Florida, and North Carolina (Henry, 1982). A California record given by Blatchley (1926: 805), considered doubtful by Henry (1982), and two Mexican records (San Lorenzo Island and Mejia Island) reported by Van Duzee (1923: 156) are here considered misidentifications. New state records are Louisiana and South Carolina.

Discussion: Henry (1982) illustrated the left and right paramere of $P$. psalliodes. In conjunction with the establishment of the lectotype, I redraw the parameres and illustrate for the first time the vesica and phallotheca (fig. 30) to help ensure the identity of this species, the type species of the genus. Specimens identified as $P$. psalliodes occurring very far west of College Station, Texas, require confirmation based on male genitalia.

Lectotype Designation: Reuter (1876) described $P$. psalliodes from Belfrage specimens taken in "Texas", but he did not indicate how many specimens he had before him. I have found seven specimens in three museums

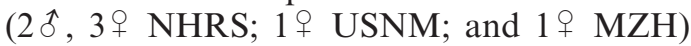

that clearly are part of the Reuter's original type series. These specimens agree with all material I have determined as $P$. psalliodes from College Station, Texas, and eastward in the United States. As a consequence, to ensure nomenclatural stability, I am designating one of two males in the NHRS as the lectotype to represent $P$. psalliodes Reuter. The remaining specimens are labeled as paralectotypes.

Type Material Examined: Lectotype $\hat{\sigma}$ (here designated): USA: Texas: Unknown Co.: collector unknown, [Belfrage Collection] (AMNH_PBI 00193960) (NHRS). PARAlECTOTYPES: Same data as for lectotype, 1 ㅇ (AMNH_PBI 00099814) (MZH); $1 \hat{\delta}$ (AMNH PBI 00193959) (NHRS); 2 (AMNH_PBI 00193957-00193958) (NHRS); $1+$ (AMNH_ PBI 00070934) (USNM).

Other SPECIMENS ExAMINED: USA: Florida: Alachua Co.: Gainesville, $29.65139^{\circ} \mathrm{N} 82.325^{\circ} \mathrm{W}$, 28 Apr 1981-29 Apr 1981, T.J. Henry, $3 \hat{\delta}$ (AMNH_PBI 00070500-00070502), 1 은 (AMNH PBI 00070499) (USNM). Gainesville, city of, $29.65139^{\circ} \mathrm{N} 82.325^{\circ} \mathrm{W}, 22$ Apr 1952, J.R. Vockeroth, $1 \hat{\delta}$ (CNC). Bay Co.: $6 \mathrm{mi} \mathrm{W}$ of Bay County line, $3 \mathrm{mi} \mathrm{W}$ of jet 231 on Rt $20,30.437^{\circ} \mathrm{N}$ $85.478^{\circ} \mathrm{W}, 9$ May 1981, T.J. Henry, Chrysoma pauciflosculosa Greene (Asteraceae), $1 \hat{\delta}$ (AMNH_ PBI 00070503) (USNM). Duval Co.: Jacksonville, $30.33194^{\circ} \mathrm{N} 81.65583^{\circ} \mathrm{W}, 23$ Jul 1926, E.D. Ball, 1 ㅇ (AMNH_PBI 00070504) (USNM). Highlands Co.: Archbold Biological Station, $27.18833^{\circ} \mathrm{N}$ 81.33778 ${ }^{\circ}$ W, 20 Apr 1981-27 Apr 1981, T.J. Henry, 5ㅇ (AMNH_PBI 00070505-00070509) (USNM). Marion Co.: Dunnellon, $29.04914^{\circ} \mathrm{N}$ $82.46093^{\circ} \mathrm{W}, 7$ Oct $1938, \quad$ P. Oman, $1 \hat{\delta}$ (AMNH_PBI 00070510) (USNM). Okaloosa Co.: $\mathrm{N}$ of Niceville, Rt. $285,30.527^{\circ} \mathrm{N} 86.468^{\circ} \mathrm{W}$, 20 Feb 2003, A.G. Wheeler, Jr., woody goldenrod, $1 \delta$ (AMNH_PBI 00070511), 2 우 (AMNH_PBI 00070512-00070513) (USNM). Seminole Co.: Sanford, $28.80055^{\circ} \mathrm{N} 81.27312^{\circ} \mathrm{W}, 1$ Mar $1926-5$ May 1927, E.D. Ball, 2 ㅇ (AMNH_PBI 00070514 00070515) (USNM). Washington Co.: Rt. 77, $1.7 \mathrm{mi}$. S of Greenhead, $29.85283^{\circ} \mathrm{N} 85.33616^{\circ} \mathrm{W}$, 18 Apr 2001, T.J. Henry and A.G. Wheeler, Jr., Chrysoma pauciflosculosa Greene (Asteraceae), 11 ㅇ (AMNH_PBI 00070516-00070526) (USNM). Louisiana: Beauregard Co.: DeRidder, $30.84631^{\circ} \mathrm{N}$

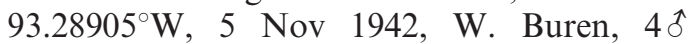
(AMNH_PBI 00070535-00070538) (USNM); 
11 Jul 1942-5 Nov 1942, W. Buren, 79 (AMNH_PBI 00070527-00070533) (USNM). North Carolina: Cumberland Co.: Fort Bragg, 15 Oct 1968, D.B. Palmer, $1 \hat{\jmath}$ (AMNH_PBI 00070534) (USNM). South Carolina: Aiken Co.: Aiken, $33.5669^{\circ} \mathrm{N} 81.7035^{\circ} \mathrm{W}, 152 \mathrm{~m}, 24$ Aug 1957, W.R. Richards, 1 s, 1 क (CNC). Lexington Co.: Peach Tree Heritage Preserve, SE of Edmond, 28 Apr 2001, A.G. Wheeler, Jr., Chrysoma pauciflosculosa Greene (Asteraceae), $3 \hat{\jmath}$ (AMNH_PBI 00070539-00070541)(USNM).

Texas: Bell Co.: Temple, USDA, ARS, Research Laboratory, $31.09^{\circ} \mathrm{N} 97.34^{\circ} \mathrm{W}, 15$ May 1987, P.E. Boldt, Baccharis sarothroides Gray (Asteraceae), 1o (AMNH_PBI 00070542) (USNM). Brazos Co.: College Station, $30.62778^{\circ} \mathrm{N} 96.33417^{\circ} \mathrm{W}, 24$ Apr 1930, H.G. Johnston, 1 우 (AMNH_PBI 00070572) (USNM); 13 Oct 1927, H.G. Johnston, 10 (AMNH_PBI 00070574) (USNM); 2 Apr 1929-16 May 1929, H.G. Johnston, 3 oै (AMNH_PBI 00070578-00070580), 3 ㅇ (AMNH_ PBI 00070575-00070577) (USNM); 20 Oct 1928, S.E. Jones, Light Trap, $2 \hat{\delta}$ (AMNH_PBI 00070581-00070582) (USNM); 15 May 1928-10 Sep 1928, H.G. Johnston, $1 \hat{\delta}$ (AMNH_PBI 00070583), 3 우 (AMNH_PBI 00070584-00070586) (USNM). College Station, $30.62778^{\circ} \mathrm{N} 96.33417^{\circ} \mathrm{W}$, 03 Oct 1928-20 Oct 1928, S.E. Jones, Gutierrezia texana Torr. \& Gray (Asteraceae), $14 \hat{\delta}$ (AMNH PBI 00070543-00070556), 15ㅇ (AMNH_PBI 00070557-00070571) (USNM); 29 Apr 1930, H.G. Johnston, $1 \hat{\delta}$ (AMNH_PBI 00070573) (USNM).

\section{Parthenicus rufus Henry}

figures 9,10

Parthenicus rufus Henry, 1982: 361 (n.sp.); Henry and Wheeler, 1988: 441 (cat.); Schuh, 1995: 181 (cat.).

Diagnosis: This species, known only from macropterous individuals, is distinguished from other species by the uniformly red body with only very small irregular pale areas on the dorsum (fig. 9, 10); the predominantly golden, sericeous setae; and the heavily redspotted legs.

Hosts: St. Johnswort, Hypericum spp.; and peelbark St. Johnswort, Hypericum fasciculatus Lam (previously Hypericaceae; now Clusiaceae) (Henry 1982). Sandhill St. Johnswort, Hypericum lloydii (Svenson) W.P. Adams, is a new host record.
Distribution: Previously known only from Collier, Highlands, Polk, and Seminole counties, Florida (Henry 1982). Franklin County is a new Florida county record. New state records are Georgia and South Carolina.

Type Material Examined: Holotype of: USA: Florida: Highlands Co.: $2 \mathrm{mi} \mathrm{E}$ of Highlands Hammock State Park on Rt 634 (Hammock Rd), [near Sebring], $27.47^{\circ} \mathrm{N}$ $81.5^{\circ} \mathrm{W}, 24$ Apr 1982, T.J. Henry and A.G. Wheeler, Jr., Hypericum fasciculatum Lam. (Clusiaceae), (AMNH_PBI 00070603). PARATYPES: USA: Florida: Collier Co.: $1.5 \mathrm{mi}$. E of Hendry Co. line, 25 Apr 1982, T.J. Henry and A.G. Wheeler, Jr., Hypericum fasciculatum Lam. (Clusiaceae), 8 우 (AMNH_PBI 00070587-00070594) (USNM). Seminole Co.: Sanford, $28.7884^{\circ} \mathrm{N} 81.2544^{\circ} \mathrm{W}, 12 \mathrm{~m}, 25 \mathrm{Aug}$ 1925-30 Aug 1925, E. D. Ball, 1 ô, 1 우 (CNC).

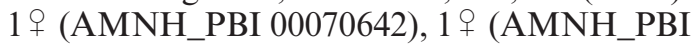
00070641) (USNM); 5 May 1927, E. D. Ball, 19 (AMNH_PBI 00070643) (USNM). Franklin Co.: $5.5 \mathrm{mi}$ W of Carrabelle Beach on Rt $98,29.804^{\circ} \mathrm{N} 84.739^{\circ} \mathrm{W}, 17$ Apr 2001, T.J. Henry and A.G. Wheeler, Jr., Hypericum sp. (Clusiaceae), $4 \hat{\delta}$ (AMNH_PBI 0007061200070615), 12 ㅇ (AMNH_PBI 00070616, AMNH_PBI 00070630-00070640) (USNM), $3 \hat{\delta}$ (AMNH_PBI 00070600-00070602), 5 우 (AMNH_PBI 00070595-00070599) (USNM). Archbold Biological Station, $27.18833^{\circ} \mathrm{N}$ 81.33778 ${ }^{\circ} \mathrm{W}, 20$ Apr 1981-27 Apr 1981, T.J. Henry, 4 ㅇ (AMNH_PBI 00070604-00070607) (USNM). Lake Placid, Archbold Biological Station, $27.188^{\circ} \mathrm{N} 81.337^{\circ} \mathrm{W}, 20$ Apr $1982-25$ Apr 1982, T.J. Henry and A.G. Wheeler,

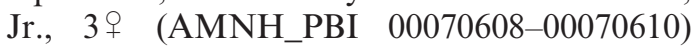
(USNM).

Other Specimens Examined: USA: Florida: Lake Co.: Hawthorne, $28.76111^{\circ} \mathrm{N}$ $81.87111^{\circ} \mathrm{W}, 2$ Jun 1961 , L.A. Kelton, $1 \hat{\jmath}$ (CNC). Georgia: Jenkins Co.: Millen, $32.80405^{\circ} \mathrm{N} 81.94928^{\circ} \mathrm{W}, 51 \mathrm{~m}, 25$ Aug 1957, J.G. Chillcott, $3 \hat{\delta}$ (CNC). McIntosh Co.: Jct I95 \& Rt. 99, near Townsend, $31.531^{\circ} \mathrm{N}$ $81.444^{\circ}$ W, 22 Apr 1984, T.J. Henry and A.G. Wheeler, Jr., Hypericum sp. (Clusiaceae), 1 웅 (AMNH_PBI 00070644) (USNM). South Carolina: Aiken Co.: Aiken, $33.5669^{\circ} \mathrm{N}$ $81.7035^{\circ} \mathrm{W}, 152 \mathrm{~m}, 12$ Jun 1957, J.R. Vockeroth, Hypericum galioides Lam. (Clusiaceae), $1 \hat{\delta}, 4$ ㅇ (CNC). Rt. 118, $1.8 \mathrm{~km} \mathrm{~S}$ of Rt. 421, 
NW of Aiken, 22 Apr 1984, A.G. Wheeler, Jr., Hypericum lloydii Svens. (Clusiaceae), $3 \hat{\delta}$ (AMNH_PBI 00070645-00070647) (USNM). Jasper Co.: N[ew] Landing, $32.51296^{\circ} \mathrm{N}$ $81.23038^{\circ} \mathrm{W}, 1$ Aug 1903, W.F. Fiske, 1 우 (AMNH_PBI 00070611) (USNM). Lexington Co.: $0.4 \mathrm{~km} \mathrm{~N}$ of S-65, $3.3 \mathrm{~km} \mathrm{E}$ of Gaston, $33.816^{\circ} \mathrm{N} 81.065^{\circ} \mathrm{W}, 5$ Jun 1999 , A.G. Wheeler, Jr., Hypericum lloydii Svens. (Clusiaceae), $2 \hat{\delta}$ (AMNH_PBI 00070649-00070650), 1 ㅇ (AMNH_PBI 00070651) (USNM). Jct. Rt. $302 \&$ S-627, $2.5 \mathrm{~km}$ NE of Edmund, 26 Apr 2003, A.G. Wheeler, Jr., Hypericum lloydii Svens. (Clusiaceae), $1 \hat{\delta} \quad\left(\mathrm{AMNH}_{-}\right.$ PBI 00070655), $3+$ (AMNH_PBI 0007065200070654) (USNM). Peach Tree Heritage Preserve, SE of Edmond, 28 Apr 2001, A.G. Wheeler, Jr., Hypericum lloydii Svens. (Clusiaceae), 10 (AMNH_PBI 00070648) (USNM).

Parthenicus sedumicola, new species

figures $11,12,31,42-59$

Holotype: USA: Arkansas: Stone Co.: Rt. 5 at Sylamore Creek Access, near jct. 9 $\& 14,35.94216^{\circ} \mathrm{N} 92.114^{\circ} \mathrm{W}, 13$ Jun 2004 , T.J. Henry and A.G. Wheeler, Jr., Sedum sp. L. (Crassulaceae), m (AMNH_PBI 00070656) (USNM).

Diagnosis: This species, known from macropterous males (figs. 11, 31) and brachypterous females (fig. 12), is distinguished from other species by the overall pale dorsum with relatively large, sparsely scattered, brown to reddish- or orange-brown spots on the hemelytra; lack of spots at the bases of the tibial spines; the long, erect, simple setae on the dorsum, intermixed with black scalelike setae on the scutellum, clavus, cuneus, and especially on the apical area of the corium; and the unique male parameres (figs. 56, 57), vesica (fig. 58), and phallotheca (fig. 59).

DESCRIPTION: Macropterous male $(N=10$; holotype in parentheses): Length 3.05-3.35 $\mathrm{mm}$ (3.25 mm), width $1.15-1.20 \mathrm{~mm}(1.15$ $\mathrm{mm})$. Head: Width $0.56-0.59 \mathrm{~mm}(0.59 \mathrm{~mm})$, vertex $\quad 0.27-0.29 \mathrm{~mm} \quad(0.29 \mathrm{~mm})$. Labium: Length $1.46-1.47 \mathrm{~mm}(1.46 \mathrm{~mm})$, extending to base of genital capsule. Antenna: Segment I, length $\quad 0.30-0.32 \mathrm{~mm} \quad(0.32 \mathrm{~mm})$; II, 1.28 $1.31 \mathrm{~mm}$ (1.23 mm); III, $0.85-0.91 \mathrm{~mm}(0.75$ $\mathrm{mm})$; IV, $0.43-0.45 \mathrm{~mm}$ (ca $0.45 \mathrm{~mm}$, curled). Pronotum: Length $0.43-0.45 \mathrm{~mm}(0.43 \mathrm{~mm})$, basal width $0.43-0.45 \mathrm{~mm}(0.43 \mathrm{~mm})$.

Brachypterous female $(N=10)$ : Length to apex of abdomen $2.15-2.30 \mathrm{~mm}$, length to apex of hemelytra $2.05-2.15 \mathrm{~mm}$, width 1.25 $1.35 \mathrm{~mm}$. Head: Width $0.58-0.59 \mathrm{~mm}$, vertex 0.34-0.35 mm. Labium: Length 1.44-1.49 mm, extending to base of ovipositor. Antenna: Segment I, length $0.29-0.34 \mathrm{~mm}$; II, $1.23-$ $1.25 \mathrm{~mm}$; III, $0.80-0.83 \mathrm{~mm}$; IV, $0.32-0.40$ $\mathrm{mm}$. Pronotum: Length $0.32-0.35 \mathrm{~mm}$, basal width $0.72-0.77 \mathrm{~mm}$. COLORATION: General coloration pale yellowish brown, with scattered orange to reddish-brown spots. Head: Yellowish brown with a yellowish-orange tinge; frons often with three or four transverse red lines. Pronotum: Pale yellowish brown on anterior half; paler whitish or ivory on posterior half, posterior area sometimes with scattered small red or orange spots; more uniformly yellowish brown in females; scutellum yellowish brown to almost green, darker yellowish brown to dusky basally. Hemelytron: Pale yellowish brown to almost dull ivory, with sparsely scattered, relatively large orange, red, or reddish-brown spots; membrane dusky brown, veins pale yellow. Ventral surface: Yellowish brown, strongly tinged with darker brown to greenish brown. Legs: Hind femora dark reddish brown, paler yellowish brown on basal third and narrowly at apex, with a few tiny black spots on distal third; middle and front femora yellowish brown, tinged with brown or reddish brown on apical third, often with a few tiny distal spots; tibiae uniformly yellowish, pale spines lacking dark spots at bases; tarsi pale yellow. TEXTURE AND VESTITURE: Dorsum impunctate, weakly shining. Head, pronotum, and hemelytra densely clothed with short, silvery, scalelike setae (most dense on head and pronotum) and black, scalelike setae on scutellum, clavus, corium, and cuneus (most dense on scutellum and inner angles of corium in males and across apical area of corium in females); intermixed with scattered long, erect, simple, pale setae on head, pronotum, and hemelytra. STRUCTURE: Males always fully macropterous (figs. 11, 31, 42). Females brachypterous, always strongly coleopteriform (figs. 12, $50,51)$, with claval suture absent; membrane 


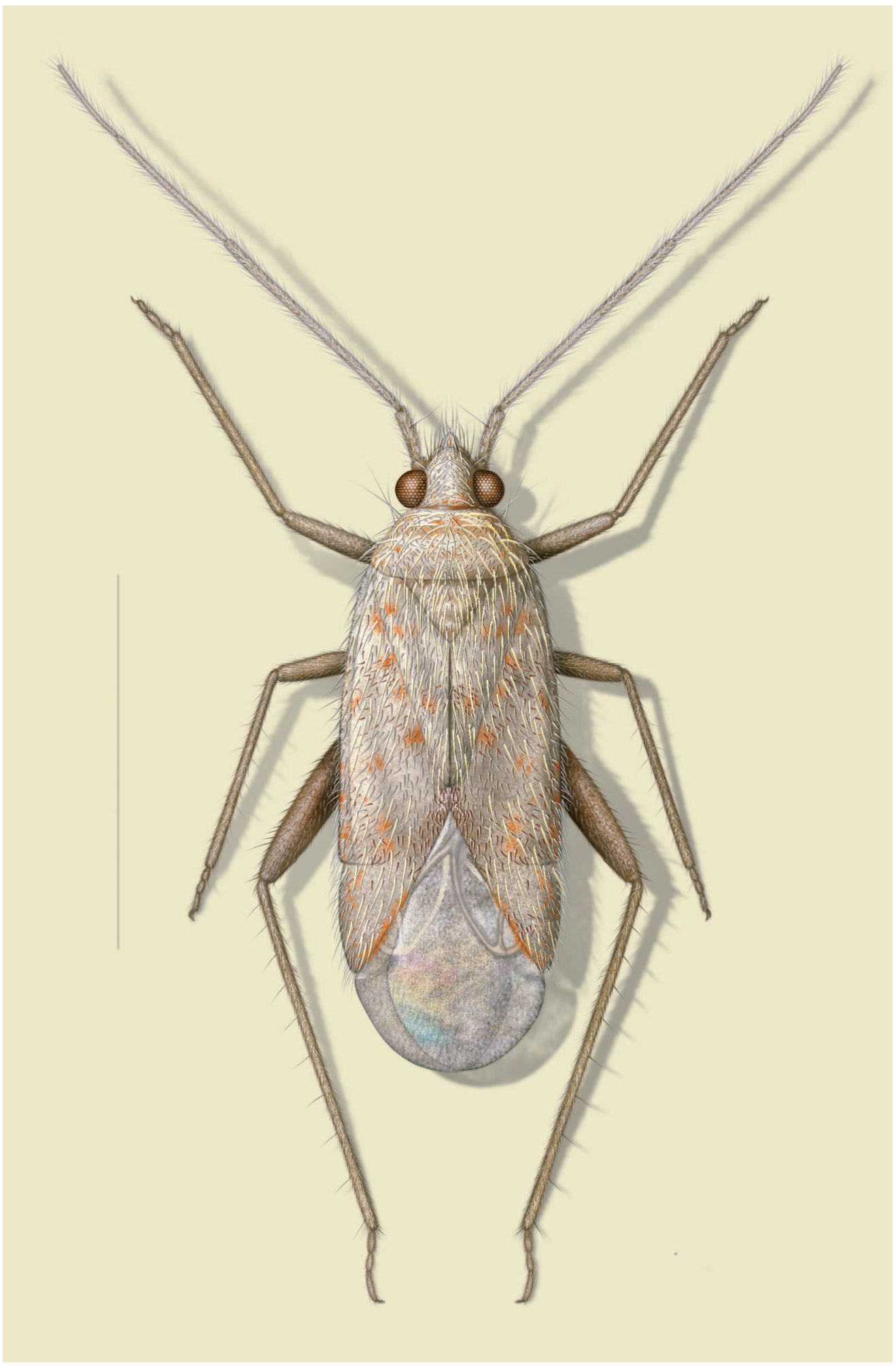

Fig. 31. Adult male of Parthenicus sedumicola, dorsal habitus. 


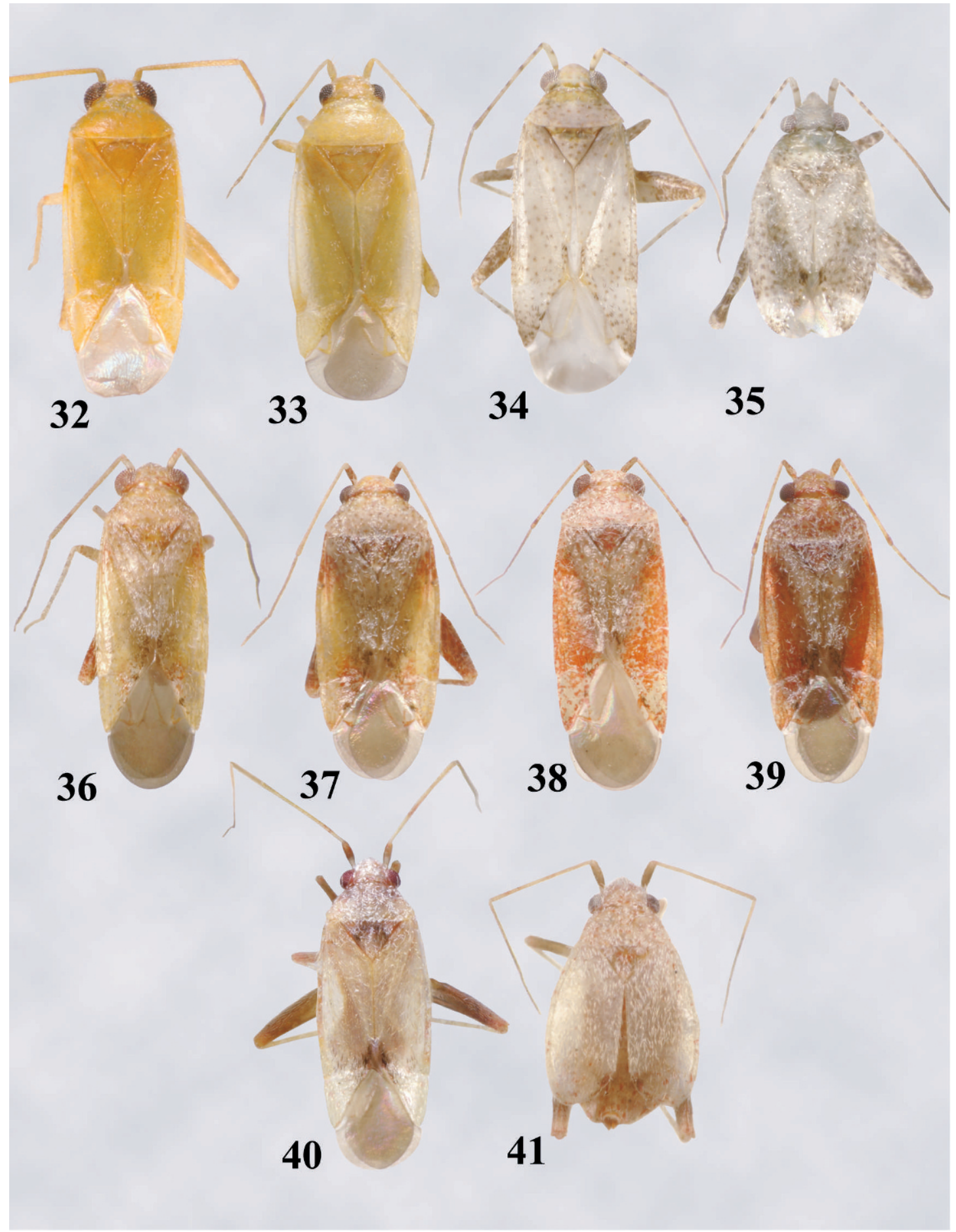

Figs. 32-41. Habitus figures of Parthenicus spp. 32, P. taxodii, male (Kentucky, Fayette Co., Lexington, University of Kentucky, 17 June year). 33, P. taxodii, female (same data as for male). 34, P. vaccini, male 
entirely absent or sometimes with only a narrow trace of membrane along posterior edge of corium and cuneus.

Male genitalia: Left paramere (fig. 56) slender and roughly C-shaped, narrowly tapering distally with a quadrate, marginally serrate process before slender apex; right paramere (fig. 57a, b) straight, stout, tapering on distal one-third, with a short, incurving, hooked process laterally; vesica (fig. 58) with two spiculi, one long, thick, subequal in diameter to secondary gonoporal process, and one short and slender; phallotheca (fig. 59) elongate, broader at base, with a lateral fingerlike process.

ETYMOLOGY: The specific epithet "sedumicola" is taken from the generic name of the host.

Hosts: Stonecrop, Sedum sp. (Crassulaceae). This species was found in abundance on its host growing on steep, dry, limestone cliffs along Rt. 5 across from Sylamore Creek Recreation Access area.

Distribution: Arkansas.

PARATypes: USA: Arkansas: Stone Co.: Rt. 5 at Sylamore Creek Access, near jct. $9 \& 14$, $35.94216^{\circ} \mathrm{N} 92.114^{\circ} \mathrm{W}, 13$ Jun 2004, T.J. Henry and A.G. Wheeler, Jr., Sedum sp. L.

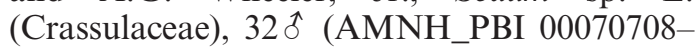
00070739), 51우 (AMNH_PBI 0007065700070707) (AMNH, CNC, USNM). Rt. 5, near Jct. 9 \& 14, near Allison, $35.93^{\circ} \mathrm{N}$ 92.11 ${ }^{\circ} \mathrm{W}, 17$ Jun 1987, T.J. Henry and A.G. Wheeler, Jr., Sedum sp. L. (Crassulaceae), $16 \hat{\jmath}$ (AMNH_PBI 00070740-00070755), 1 우 (AMNH_PBI 00070756), 70 우 (AMNH_PBI 00070757-00070826) (AMNH, CNC, USNM).

\section{Parthenicus taxodii Knight}

figures 32,33

Parthenicus taxodii Knight, 1941: 76 (n.sp.); Froeschner, 1949: 166 (note, key); Carvalho,
1958: 124 (cat.); Henry, 1982: 363 (descrip., distr., host, key, figs.); Snodgrass et al., 1984: 853 (list, host); Henry and Wheeler, 1988: 441 (cat.); Schuh, 1995: 181 (cat.); Henry et al., 2005: 61 (distr., host).

Diagnosis: This species, known only from macropterous specimens, is distinguished from other species by the small size; uniformly yellow coloration (figs. 32, 33), often with a faint orange tinge; silvery sericeous pubescence on the dorsum; and the pallid tibial spines lacking spots at the bases.

Host: Bald cypress, Taxodium distichum (L.) Rich. (Taxodiaceae) (Knight, 1941).

Distribution: Originally described from Illinois (Knight, 1941) and later reported from Missouri (Froeschner 1949), Florida, Kentucky, and Mississippi (Henry, 1982; Henry and Wheeler, 1988; Henry et al., 2005). New state records are Arkansas, Maryland, and Tennessee.

Type Material Examined: Paratypes: USA: Illinois: Alexander Co.: Cairo, $37.00528^{\circ} \mathrm{N}$ $89.17639^{\circ} \mathrm{W}, 27$ Jul 1930, Knight and Ross, Taxodium distichum L. (Taxodiaceae), $1 \hat{\sigma}$ (AMNH_PBI 00070895), 1 ㅇ (AMNH_PBI 00070894) (USNM). Champaign Co.: Urbana, $40.11056^{\circ} \mathrm{N} 88.20722^{\circ} \mathrm{W}, 28$ Aug 1917, H.H. Knight, $1+$ (AMNH_PBI 00070893) (USNM). Pulaski Co.: Karnak, $37.2936^{\circ}$ N 88.97535 W, 26 Jul 1930, H.H. Knight, Taxodium distichum L. (Taxodiaceae), 4t (AMNH_PBI 00070844 00070847), 6 ㅇ (AMNH_PBI 00070848-00070853) (USNM); 26 Jul 1930, Knight and Ross, Taxodium distichum L. (Taxodiaceae), $9 \hat{\delta}$ (AMNH_PBI 00070827-00070835), 8ㅇ (AMNH_PBI 00070836 00070843) (USNM). Union Co.: Jonesboro, $37.45172^{\circ} \mathrm{N} 89.26814^{\circ} \mathrm{W}, 2$ Aug 1932, H. L. Dozier, Taxodium distichum L. (Taxodiaceae), $2 \hat{\jmath}$ (AMNH_PBI 00070856, AMNH_PBI 00070857), 2 ( (AMNH_PBI 00070854-00070855) (USNM).

(New York, Suffolk Co., nr. Quoque, 29 August 1981). 35, P. vaccini, female (Michigan, Marquette Co., Rt. 28, E of Harvey, 25 July 1991). 36, P. weemsi, male (pale form) (Florida, Highlands Co., 12 mi S of Lake Placid, 23 April 1982). 37, P. weemsi, female (pale form) (Florida, Highlands Co., Rt. 70, 2 mi W of Rt. 27, nr Archbold Biol. Stn., 30 April 1982). 38, P. weemsi, male (dark form) (Florida, Saint Lucie Co., Port Saint Lucie, 6 February 1989). 39, P. weemsi, female (dark form) (Florida, Highlands Co., Rt. 27, 9.5 km NNW of Lake Placid, 6 February 2000). 40, P. wheeleri, male (Oklahoma, Garvin Co., Rt. 76, 4 km N of Stephens Co. line, NW of Purdy, 20 May 2001). 41, P. wheeleri, female (Texas, Mills Co., Rt. 16, 9 km SW of Goldthwaite, 30 May 2001). 

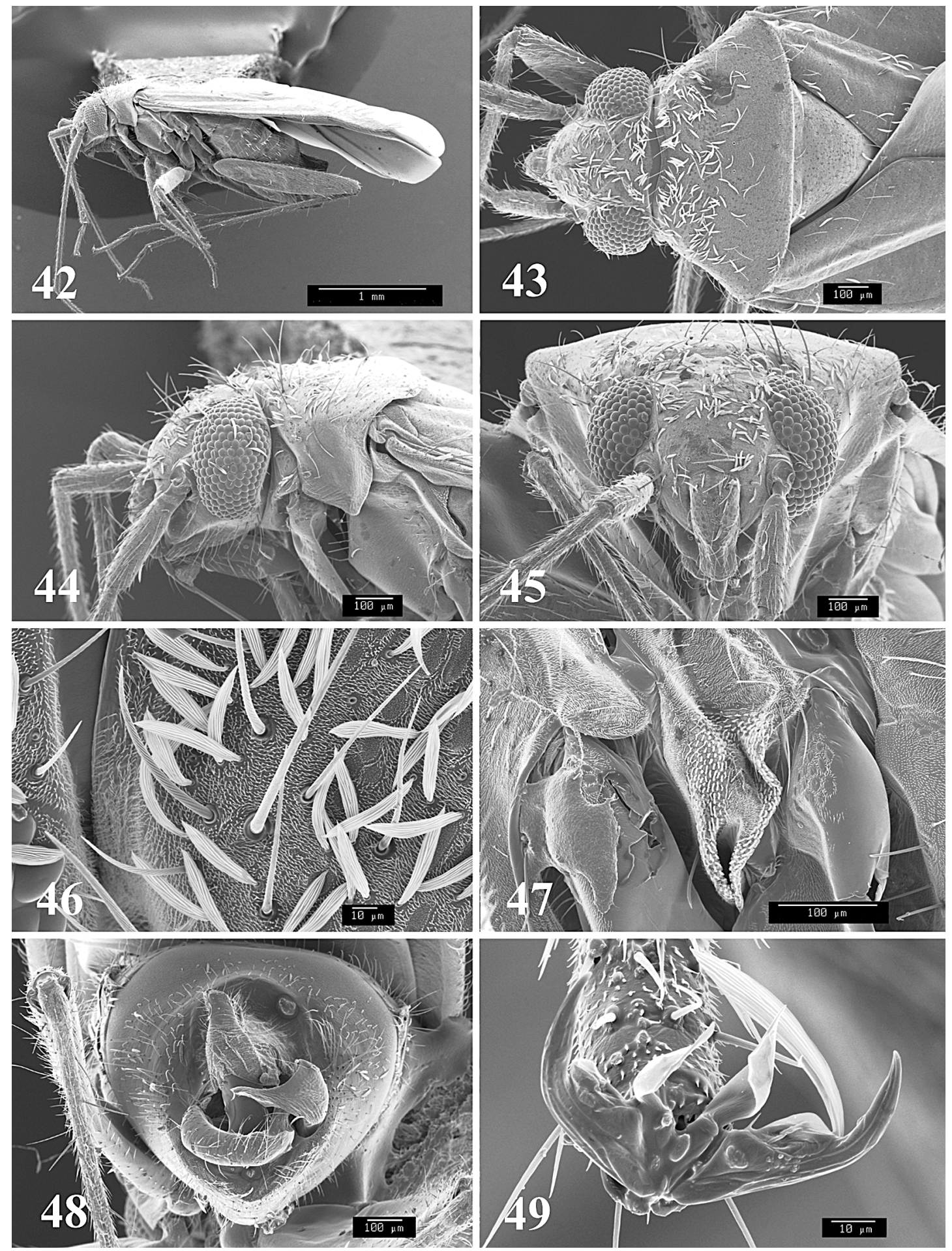

Figs. 42-49. Scanning electron photomicrographs of adult male Parthenicus sedumicola. 42, Lateral aspect $(28.4 \times)$. 43, Head and pronotum, dorsal aspect $(79.0 \times)$. 44, Head and pronotum, lateral aspect $(97.5 \times) .45$, Head, frontal aspect $(102 \times)$. 46, Scalelike setae on head $(660 \times) .47$, Ostiolar evaporative auricle $(258 \times)$. 48, Genital capsule, caudal aspect $(92.5 \times)$. 49, Claw $(1150 \times)$. 

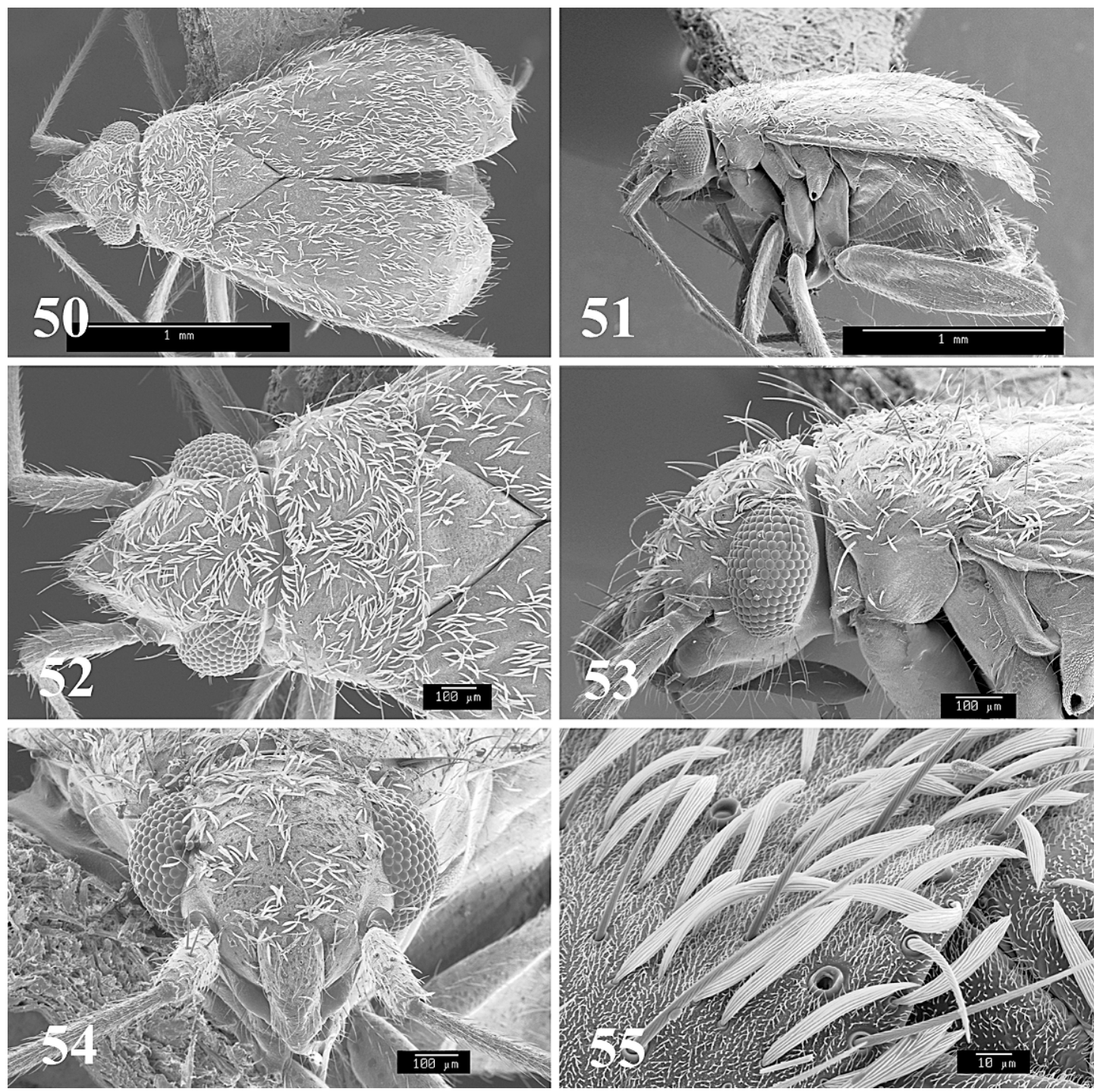

Figs. 50-55. Scanning electron photomicrographs of adult female Parthenicus sedumicola. 50, Dorsal aspect $(41.2 \times)$. 51, Lateral aspect $(41.1 \times)$. 52, Head and pronotum, dorsal aspect $(79.5 \times)$. 53, Head and pronotum, lateral aspect $(106 \times)$. 54, Head, frontal aspect $(97.0 \times)$. 55, Scalelike setae on head $(825 \times)$.

Other Specimens Examined: USA: Arkansas: Chicot Co.: Lake Village, $33.32873^{\circ} \mathrm{N}$ 91.2817 ${ }^{\circ}$ W, 9 Jun 1987, T.J. Henry and A.G. Wheeler, Jr., Taxodium distichum L. (Taxodiaceae), 8 우 (AMNH_PBI 00070858-00070865) (USNM). Florida: Liberty Co.: 3 mi. S of Bristol, County Road $379,30.388^{\circ} \mathrm{N} 84.965^{\circ} \mathrm{W}, 7$ May 1981, T.J. Henry, Taxodium distichum L. (Taxodiaceae), 2犬̂े (AMNH_PBI 0007086800070869), 2 + (AMNH_PBI 00070866-00070867)
(USNM). Illinois: Ft. Defiance, Cairo Point, $36.98516^{\circ} \mathrm{N} 89.13466^{\circ} \mathrm{E}, 20$ Jun 2001, T.J. Henry and A.G. Wheeler, Jr, Taxodium distichum L. (Taxodiaceae), 7 우 (AMNH_PBI 00070870-00070876) (USNM). Kentucky: Bullitt Co.: Bernheim Arboretum and Research Forest, $37.91833^{\circ} \mathrm{N} 85.66716^{\circ} \mathrm{W}$, 22 Jun 2001, T.J. Henry and A.G. Wheeler, Jr., Taxodium distichum L. (Taxodiaceae), $2 \hat{\delta}$ (AMNH_PBI 00070879-00070880), 3ㅇ (AMNH_ PBI 00070881-00070883) (USNM). Calloway Co.: 


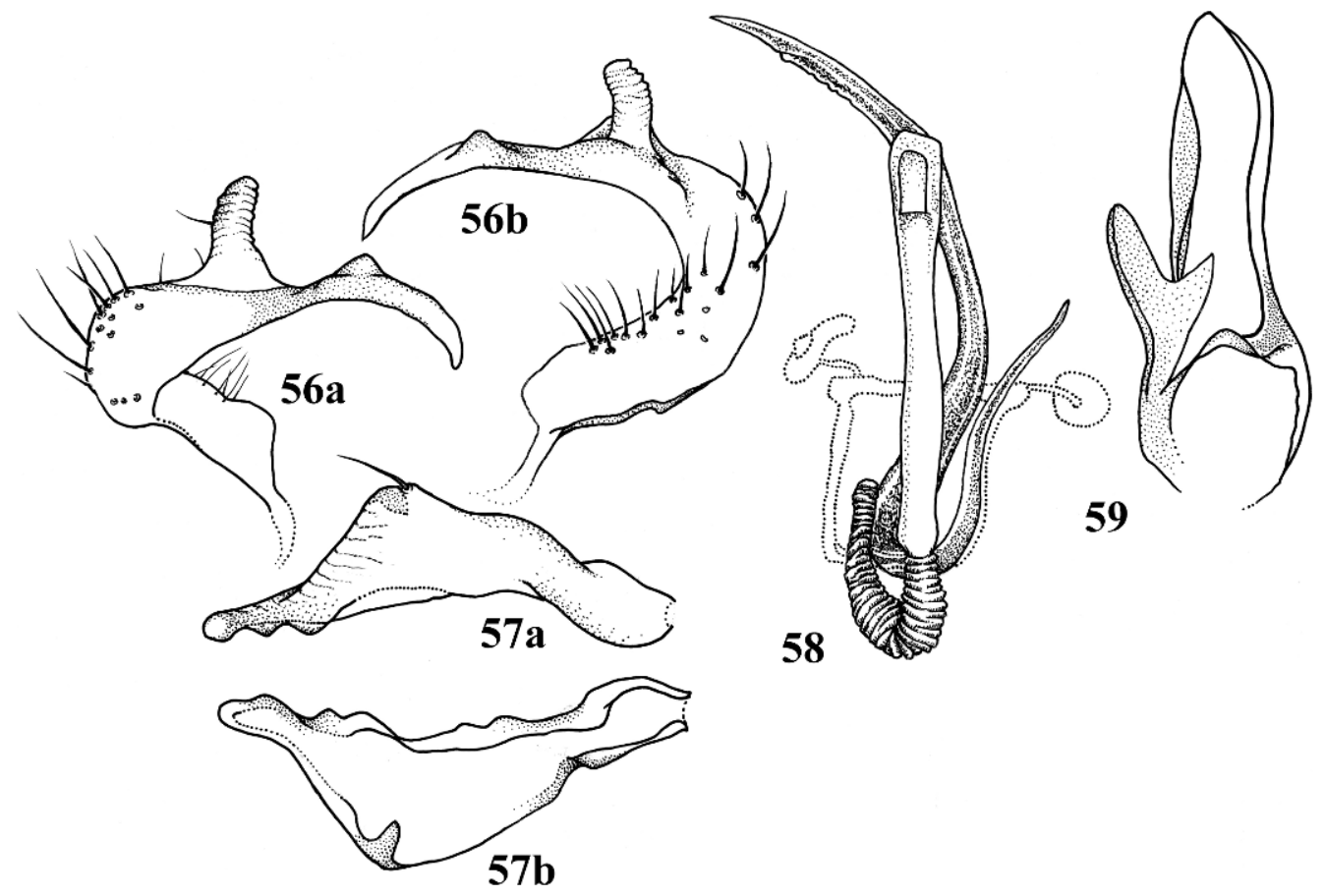

Figs. 56-59. Male genitalia of Parthenicus sedumicola. 56, Left paramere (a, dorsal aspect; b, caudal aspect). 57, Right paramere (a, caudal aspect; b, anterior aspect). 58, Vesica. 59, Phallotheca.

Murray, Calloway County Park, $36.60933^{\circ} \mathrm{N}$ $88.30716^{\circ} \mathrm{E}, 19$ Jun 2001, T.J. Henry and A.G. Wheeler, Jr., Taxodium distichum L. (Taxodiaceae), $1 \delta$ (AMNH_PBI 00070877), 1 우 (AMNH_PBI 00070878) (USNM). Fayette Co.: Lexington, University of Kentucky Campus, $38.03483^{\circ} \mathrm{N}$ $84.5025^{\circ} \mathrm{W}, 17$ Jun 2001, T.J. Henry and A.G. Wheeler, Jr., Taxodium distichum L. (Taxodiaceae), 17 $\delta$ (AMNH_PBI 00070897-00070913), 20 우 (AMNH_PBI 00070914-00070933) (USNM). Maryland: St. Mary's Co.: Point Lookout, $39.07678^{\circ} \mathrm{N} 76.59385^{\circ} \mathrm{W}, 20$ Jul 1985, T.J. Henry and A.G. Wheeler, Jr., Taxodium distichum L. (Taxodiaceae), 69 (AMNH_PBI 00070884 00070889) (USNM). Mississippi: Washington Co.: Greenville, $33.4101^{\circ} \mathrm{N} 91.06177^{\circ} \mathrm{W}, 19$ Jul 1982 , G.L. Snodgrass, 1 ㅇ (AMNH_PBI 00070890) (USNM). Stoneville, $33.42401^{\circ} \mathrm{N} 90.9151^{\circ} \mathrm{W}, 14$ Jul 1982, G.L. Snodgrass, Gossypium sp. L. (Malvaceae), 1 우 (AMNH_PBI 00070891) (USNM). Missouri: Dunklin Co.: Holcomb, 36.39923N $90.0237^{\circ}$ W, 23 Aug 1942, R.C. Froeschner, 1 우 (AMNH_PBI 00070896) (USNM). Tennessee: Tipton Co.: $3 \mathrm{mi} \mathrm{N}$ Covington, $35.607^{\circ} \mathrm{N}$ $89.646^{\circ} \mathrm{W}, 2$ Jun 1985, T.J. Henry and A.G.
Wheeler, Jr., Taxodium distichum L. (Taxodiaceae), $1 \hat{\jmath}$ (AMNH_PBI 00070892)(USNM).

\section{Parthenicus vaccini (Van Duzee)}

figures 34,35

Psallus vaccini Van Duzee, 1915: 117 (n.sp.).

Parthenicus vaccini: Van Duzee, 1916: 45 (list), 1917: 401 (cat.), 1918: 30 (key); Knight, 1918: 43 (fig. 17), 1923: 499 (descrip., key), 1941: 21 (fig. 54); Blatchley, 1926: 805 (descrip., note, key); Carvalho, 1958: 124 (cat.); Henry, 1978: 546 (note, host), 1982: 364 (descrip., distr., hosts, key, figs.); Henry and Wheeler, 1988: 442 (cat.); Schuh, 1995: 181 (cat.); Roble and Hoffman, 2000: 44 (note, hosts, distr.).

Diagnosis: This species is recognized by the overall gray to grayish-green coloration (figs. 34, 35), with numerous fuscous spots on the dorsum, legs, and antennae; and the long rostrum that extends to near the base of the genital capsule. Males (fig. 34) are always macropterous, whereas females are known from both macropterous and subbrachypterous (fig. 35) or short-winged indi- 
viduals with abbreviated hemelytral membranes.

Hosts: As Henry (1978) pointed out, the specific epithet vaccini, named for the host genus Vaccinium (Ericaceae), is a misnomer. The true hosts for this species are Pinebarren goldenheather, Hudsonia ericoides L. (Cistaceae); woolly beachheather, H. tomentosa Nutt.; and beach pinweed, Lechia maritima Leggett (Cistaceae) (Henry, 1978, 1982), all specialized plants that grow in sandy pinebarren habitats along the East Coast and around certain areas of the Great Lakes.

DisTRIBUTION: Originally described from Massachusetts (Van Duzee, 1915) and later reported from New York (Knight, 1923), Florida (Blatchley, 1926), New Jersey (Henry, 1978), and Virginia (Roble and Hoffman, 2000). New state records are Michigan and New Hampshire.

Type Material Examined: Paratype: USA: Massachusetts: Essex Co.: Ipswich, $42.67917^{\circ} \mathrm{N} 70.84167^{\circ} \mathrm{W}, 22$ May 1909, E.P. Van Duzee, $1 \delta$ (CNC).

Other Specimens Examined: USA: Massachusetts: Michigan: Alpena Co.: Lakewood, $45.19085^{\circ} \mathrm{N} 83.43387^{\circ} \mathrm{W}, 200 \mathrm{~m}, 28 \mathrm{Jul} 1960$, Kelton and Whitney, Hudsonia sp. (Cistaceae), 26ㅅ․ 12으 (CNC); 18 Aug 1960, Kelton and Whitney, Hudsonia sp. (Cistaceae), 50^, 49우 (CNC). Marquette Co.: Rt. 28, E of Harvey, $46.489^{\circ} \mathrm{N} 87.332^{\circ} \mathrm{W}, 25$ Jul 1991, T.J. Henry and A.G. Wheeler, Jr., Hudsonia sp. (Cistaceae), 7 के (AMNH_PBI 00070943-00070949), 9우 (AMNH_ PBI 00070935-00070943) (USNM). New Hampshire: Rockingham Co.: Seabrook, Rt. 1A along dunes, 28 Jun 1989, T.J. Henry and A. G. Wheeler, Jr., Hudsonia tomentosa Nutt. (Cistaceae), 58े (AMNH_PBI 00070950-00070954), 2ㅇ (AMNH_ PBI 00070955-00070956) (USNM). New Jersey: Ocean Co.: Lakehurst, $40.01444^{\circ} \mathrm{N} 74.31111^{\circ} \mathrm{W}$, 18 m, 30 Aug 1915, collector unknown, Hudsonia sp. (Cistaceae), $1 \hat{\delta}$ (CNC); 1 Aug 1915, H. G. Barber, Hudsonia sp. (Cistaceae), $1+$ (AMNH_PBI 00070957) (USNM). Rt. 37, near Tom's River, $39.9628^{\circ} \mathrm{N} 74.1921^{\circ} \mathrm{W}, 13$ Jun 1977 , T.J. Henry and A.G. Wheeler, Jr., Hudsonia ericoides L. (Cistaceae), $1 \hat{\delta}$ (AMNH_PBI 00070959) Hudsonia ericoides L. (Cistaceae), 11 ㅇ (AMNH_PBI 0007096000070970) (USNM). Seaside Park, $39.92679^{\circ} \mathrm{N}$ $74.07708^{\circ} \mathrm{W}, 1$ Jul 1915 , Weiss and West, $1 \delta^{\hat{\sigma}}$ (AMNH_PBI 00070958) (USNM). New York:
Suffolk Co.: Mattituck, Long Island, $40.99121^{\circ} \mathrm{N}$ $72.5342^{\circ} \mathrm{W}, 7$ Oct $1947, \mathrm{R}$. Latham, $1 \hat{\jmath}$ (AMNH_PBI 00070971) (USNM). Near Quogue, $40.82305^{\circ} \mathrm{N} 72.60944^{\circ} \mathrm{W}, 5 \mathrm{~m}, 29$ Aug 1981 , T.J. Henry and A.G. Wheeler, Jr., Hudsonia tomentosa L. (Cistaceae), $15 \hat{\jmath}$ (AMNH_PBI 00070972-00070986), 1 ㅇ (AMNH_PBI 00070987) (USNM). Near Yaphank, $40.83666^{\circ} \mathrm{N} 40.83666^{\circ} \mathrm{W}$, 13 m, 29 Aug 1981, T.J. Henry and A.G. Wheeler, Jr., Hudsonia tomentosa Nutt. (Cistaceae), $8 \hat{\delta}$ (AMNH_PBI 00070988-00070995), 1 우 (AMNH_ PBI 00070996) (USNM).

\section{Parthenicus weemsi Henry figures 36-39}

Parthenicus weemsi Henry, 1982: 364 (n.sp.); Henry and Wheeler, 1988: 442 (cat.); Schuh, 1995: 181 (cat.).

Diagnosis: This species, known only from macropterous individuals, is distinguished from other species by the usually testaceous to golden-yellow color (fig. 36, 37), with brown and/or red spots on the pronotum and the base and apex of the corium, and the numerous red spots on the legs. Darker specimens sometimes also are present with the corium and cuneus becoming much darker red to reddish brown. These might be confused with $P$. rufus, but the pronotum and clavus remain pale, with only a few red spots, and they are densely covered in white, scalelike setae.

Host: Sand heath, Ceratiola ericoides Michx. (Empetraceae).

Distribution: Previously known only from Highlands and Putnam counties, Florida (Henry, 1982). New Florida county records are Alachua, Lake, Levy, Walton, and Saint Lucie counties.

Type Material Examined: Holotype $\hat{\sigma}$ : USA: Florida: Highlands Co., Rt 70, 2 mi W of Rt 27, near Archbold Biological Station, $27.2^{\circ} \mathrm{N} 81.37^{\circ} \mathrm{W}, 20$ Apr 1982, T.J. Henry and A.G. Wheeler, Jr, Ceratiola ericoides Michx. (Empetraceae), (AMNH_PBI 00070997). PARATYPES: USA: Florida: Highlands Co., Rt 70, $2 \mathrm{mi} \mathrm{W}$ of Rt 27, near Archbold Biological Station, $27.2^{\circ} \mathrm{N} 81.37^{\circ} \mathrm{W}, 20$ Apr 1982 , T.J. Henry and A.G. Wheeler, Jr., Ceratiola ericoides Michx. (Empetraceae), $6 \hat{\jmath}$ (AMNH_ PBI 00070998-00071003), 20 우 (AMNH_ 
PBI 00071004-00071023) (USNM). Putnam Co.: unknown locality, $29.62^{\circ} \mathrm{N} 81.73^{\circ} \mathrm{W}$, 6 Jul 1956, H.V. Weems, Jr., Ceratiola ericoides Michx. (Empetraceae), 5 oे (AMNH PBI 00071024-00071028), 3 ㅇ (AMNH_PBI 00071029-00071031) (USNM).

Other SPecimens Examined: USA: Florida: Alachua Co.: Archer, $29.52997^{\circ} \mathrm{N} 82.519^{\circ} \mathrm{W}$, 28 m, 3 May 1961, L.A. Kelton, rosemary, 11 $\hat{\delta}$, 15 ㅇ (CNC). Rt. 24, $2 \mathrm{mi}$. W of Archer, $29.50866^{\circ} \mathrm{N} 82.552^{\circ} \mathrm{W}, 15$ Apr 2001, T.J. Henry and A.G. Wheeler, Jr., Ceratiola ericoides Michx. (Empetraceae), $3 \delta$ (AMNH_PBI 00071032 00071034), 6 우 (AMNH_PBI 00071035-00071040) (USNM). Highlands Co.: $12 \mathrm{mi}$ S of Lake Placid, $27.118^{\circ} \mathrm{N} 81.363^{\circ} \mathrm{W}, 23 \mathrm{Apr} 1982$, T.J.Henryand A.G. Wheeler, Jr, Ceratiola ericoides Michx. (Empetraceae), 1 to (AMNH_PBI 00071048), 4우 (AMNH_PBI $00071044-00071047)$ (USNM). $9.5 \mathrm{~km} \mathrm{NNW}$ of Lake Placid on Rt $27,27.48^{\circ} \mathrm{N} 81.48^{\circ} \mathrm{W}, 6$ Feb 2000, A.G. Wheeler, Jr., Ceratiola ericoides Michx. (Empetraceae), $8+$ (AMNH_PBI 00071049-00071 056)(USNM). Rt 70, S of Lake Placid, near Archbold Biological Station, $27.2^{\circ} \mathrm{N} 81.31^{\circ} \mathrm{W}, 25$ Apr 1984, T.J. Henry and A.G. Wheeler, Jr., Ceratiola ericoides Michx. (Empetraceae), 3 (AMNH_PBI 0007104100071043) (USNM). Near Polk County line, ca. $6.5 \mathrm{~km}$ NW of Avon Park, 27.645 N 81.529 $\mathrm{W}, 25$ Feb 2002, A.G. Wheeler, Jr., Ceratiola ericoides Michx. (Empetraceae), $1 \delta$ (AMNH_PBI 00071068) (USNM). Levy Co.: $3 \mathrm{mi}$ E of Archer, Rt. 17, 23 Apr 1984, T.J. Henry and A.G. Wheeler, Jr, Ceratiola ericoides Michx. (Empetraceae), 2 을 (AMNH_PBI 00071058-00071059) (USNM). Polk Co.: 5.2 km S of Frostproof, Rt. 17, $27.698^{\circ} \mathrm{N} 81.53^{\circ} \mathrm{W}, 5$ Feb 2003, A.G. Wheeler, Jr., Ceratiola ericoides Michx. (Empetraceae), $1 \delta^{\hat{\sigma}}$ (AMNH_PBI 00071069), 2 ㅇ (AMNH_PBI 00071070-00071071) (USNM). St. Lucie Co.: Port Saint Lucie, $27.293^{\circ} \mathrm{N} 80.35^{\circ} \mathrm{W}, 6 \mathrm{Feb}$ 1989, K.L. Hibbard, Ceratiola ericoides Michx. (Empetraceae), $3 \hat{\delta}$ (AMNH_PBI 0007106000071062), 1 ㅇ (AMNH_PBI 00071063) (USNM). Volusia Co.: Rt 19, $7 \mathrm{mi} \mathrm{S}$ from jet of Rt 40, $7 \mathrm{mi}$ $\mathrm{N}$ of Altoona, $29.065^{\circ} \mathrm{N} 81.634^{\circ} \mathrm{W}, 24$ Apr 1984 , T.J. Henry and A.G. Wheeler, Jr, Ceratiola ericoides Michx. (Empetraceae), 1 우 (AMNH_PBI 00071057) (USNM). Walton Co.: S Sandestine Blvd, $10 \mathrm{mi}$ E of Destin, $30.37^{\circ} \mathrm{N} 86.33^{\circ} \mathrm{W}, 3 \mathrm{~m}, 1$ Mar 1999-2 Mar 1999, A.G. Wheeler, Jr., Ceratiola ericoides Michx. (Empetraceae), 20 (AMNH_PBI 00071064-00071065), 2ㅇ (AMNH_ PBI 00071066-00071067) (USNM).

\section{Parthenicus wheeleri, new species}

figures 40, 41, 60-77

Holotype $\hat{\delta}$ : USA: Oklahoma: Dewey Co.: $0.2 \mathrm{~km} \mathrm{~W}$ of jet. Rt. $270 / 281,6.8 \mathrm{~km}$ SE of Oakwood, 21 May 2001, A.G. Wheeler, Jr., Eragrostis curvula Schrad. (Poaceae) (AMNH_PBI 00071072) (USNM).

Diagnosis: This species, known from macropterous males (figs. 11, 60) and strongly brachypterous, coleopteriform females (figs. $12,68,69)$, is distinguished by the pale testaceous dorsum (figs. 11, 12), occasionally with a few small red speckles on the head, pronotum, and hemelytra, especially in females, contrasted by the brown first antennal segment, scutellum, and hind femur; the lack of spots at the bases of the tibial spines on the front and middle legs and frequently on the hind legs (or with only very faint reddish spots on some basal spines); and distinctive male parameres (figs. 74, 75), vesica (fig. 76), and phallotheca (fig. 77).

DESCRIPTION: Macropterous male $(N=10$; holotype in parentheses): Length to apex of membrane $3.05-3.45 \mathrm{~mm}$ (3.15 mm), length to base of cuneus $1.95-2.35 \mathrm{~mm}(2.21 \mathrm{~mm})$, width $1.20-1.25 \mathrm{~mm}(1.15 \mathrm{~mm})$. Head: Length $0.48-0.53 \mathrm{~mm}(0.48 \mathrm{~mm})$, width across eyes $0.61-0.64 \mathrm{~mm} \quad(0.61 \mathrm{~mm})$, width of vertex $0.30-0.34 \mathrm{~mm} \quad(0.30 \mathrm{~mm})$. Labium: Length $1.33-1.39 \mathrm{~mm}(1.39 \mathrm{~mm})$, extending beyond metacoxae to base of genital capsule. Antenna: Segment I, length $0.27-0.32 \mathrm{~mm}(0.27 \mathrm{~mm})$; II, $1.09-1.17 \mathrm{~mm}$ (1.12 mm); III, 0.75-0.88 $\mathrm{mm}(0.80 \mathrm{~mm})$; IV, $0.45-0.50 \mathrm{~mm}(0.46 \mathrm{~mm})$. Pronotum: Length $0.38-0.43 \mathrm{~mm}(0.42 \mathrm{~mm})$, basal width $0.88-0.93 \mathrm{~mm}(0.91 \mathrm{~mm})$. Brachypterous female: $(N=10)$ : Length to apex of abdomen 2.15-2.45 mm, length to base of cuneus $1.75-1.90 \mathrm{~mm}$, width $1.22-1.31 \mathrm{~mm}$. Head: Length $0.43-0.45 \mathrm{~mm}$, width across eyes $0.61-0.62 \mathrm{~mm}$, width of vertex 0.34 $0.37 \mathrm{~mm}$. Labium: Length $1.30-1.41 \mathrm{~mm}$, extending to base of ovipositor or slightly beyond. Antenna: Segment I, length 0.29$0.30 \mathrm{~mm}$; II, $0.96-0.99 \mathrm{~mm}$; III, 0.75-0.77 $\mathrm{mm} ; 0.78-0.82 \mathrm{~mm}$. Pronotum: Length $0.32-$ $0.34 \mathrm{~mm}$, basal width $0.78-0.82 \mathrm{~mm}$. COLORATION: Overall coloration pale yellowish brown, with only a few scattered red spots on dorsum. Head: Pale yellowish brown, vertex 
between eyes, clypeus, and sides with scattered red spots; frons with five slender, transverse red lines on each side. Pronotum: Pale yellowish brown, area across calli more dusky brown, evenly scattered with diffuse red spots; scutellum pale yellowish brown with only basal angles brown to more completely brown with only the apex paler. Hemelytron: Pale yellowish brown, with red spots ranging from only along borders of lateral margins, cuneus, and sutures along clavus to more evenly distributed; membrane in macropters uniformly smoky brown to fumate but not black, veins pallid, sometimes with a few tiny red spots. Ventral surface: Pale yellowish brown, strongly accented with red along margins of sutures on thorax to dark brown with less red in some specimens; abdomen with scattered irregular red spots and a diffuse, broken red line laterally. Legs: Front and middle femora pale yellowish brown with a few red or brown spots on distal third; hind femur dark brown on distal two-thirds with a few tiny red or darker brown spots; tibiae pale yellowish brown without spots at bases of pale spines; tarsi pale yellowish brown; claws dark brown or fuscous. TEXTURE AND VESTITURE: Impunctate and semishiny. Head, pronotum, and scutellum with long, slender, white simple setae, densely intermixed with silvery sericeous and scalelike setae; hemelytra with recumbent pale yellowishbrown simple setae, intermixed with relatively slender pale yellowish brown to whitish scalelike setae on clavus and corium, with black scalelike setae along basal and inner margin of cuneus and distal margin of corium bordering membrane. STRUCTURE: Males fully macropterous (fig. 11) with well-developed hemelytra. All known females (fig. 12) with hemelytron abbreviated, coleopteriform, with the claval suture absent, forming a smooth beetlelike "elytra"; cuneus greatly shortened and rounded apically; wing membrane ranging from only a narrow strip to absent.

Male genitalia: Left paramere (fig. 74) Cshaped with base much broader than slender apical third; right paramere (fig. 75) relatively stout, straight with lateral margins narrowly incurved and apex with a lateral hook; vesica (fig. 76) with one complex spiculum having multiple spines and an elongate, slender apical process; phallotheca (fig. 77) dome-shaped with a broad distal opening.

ETYMOLOGY: I have the pleasure of naming yet another new species after Dr. Alfred George Wheeler, Jr., collector of the entire series of this plant bug. Dr. Wheeler's tenacity for seeking and collecting rare, uncommon, and new Heteroptera in unique habitats on unusual hosts continues to show that we have much to learn about many areas of the United States.

Hosts: Weeping lovegrass, Eragrostis curvula (Schrad) Nees (Poeaceae); sand lovegrass, E. trichodes (Nutt.) Wood; Lindheimer's muhly, Muhlenbergia lindheimeri Hitchc. (Poaceae); seep muhly, M. reverchonii Vasey and Schribn. (Poaceae).

Distribution: Oklahoma and Texas.

PARATYPes: USA: Oklahoma: Blaine Co.: Rt. 3/33/270, $13 \mathrm{~km} \mathrm{~W}$ of Watonga, 23 Apr 2002, A.G. Wheeler, Jr., Eragrostis curvula Schrad. (Poaceae), $1 \hat{\delta} \quad\left(\mathrm{AMNH}_{-}\right.$ PBI 00071075) (USNM). Dewey Co.: $0.2 \mathrm{~km}$ W of jct. Rt. 270/281, $6.8 \mathrm{~km}$ SE of Oakwood, 21 May 2001, A.G. Wheeler, Jr., Eragrostis curvula Schrad. (Poaceae), $27 \hat{\jmath}$ (AMNH_PBI 00071073, AMNH_PBI 00071076-00071101), 25 우 (AMNH_PBI 00071102-00071126) (AMNH, CNC, USNM). Rts. 270/281, $6.8 \mathrm{~km}$ SE of Oakwood, 20 May 2003, A.G. Wheeler, Jr., Eragrostis curvula Schrad. (Poaceae), 1 ㅇ (AMNH_PBI 00071185) (USNM). Garvin Co.: Rt. 76, $4.0 \mathrm{~km} \mathrm{~N}$ of Stephens Co. line (Purdy), 28 Apr 2000-30 Apr 2000, A.G. Wheeler, Jr., Eragrostis trichodes Nutt. (Poaceae), $8 \hat{\jmath}$ (AMNH_PBI 0007112700071134), 6우요 (AMNH_PBI 0007113500071140) (USNM). Rt. $76,4.0 \mathrm{~km} \mathrm{~N}$ of Stephens Co. line, NW of Purdy, 20 May 2001, A.G. Wheeler, Jr., Muhlenbergia reverchonii Vasey \& Scribn. (Poaceae), $1 \hat{\sigma}$ (AMNH_PBI 00071146), 3 ㅇ (AMNH_PBI 00071143-00071145) Muhlenbergia reverchonii A.S. Hitchc. (Poaceae), $2 \hat{\delta}$ (AMNH_PBI 00071141, AMNH_PBI 00071142) (USNM). Rt. 76, Purdy, 28 May 2003, A.G. Wheeler, Jr., Muhlenbergia reverchonii Vasey \&Scribn. (Poaceae), 1 ㅇ (AMNH_PBI 00071147) (USNM). Johnston Co.: Rt. 99/377, $1.0 \mathrm{~km} \mathrm{~S}$ of Connerville, 11 Apr 2002, A.G. Wheeler, Jr., Muhlenbergia reverchonii Vasey \& Scribn. (Poaceae), 1 § (AMNH_PBI 00071148) (USNM). Murray 

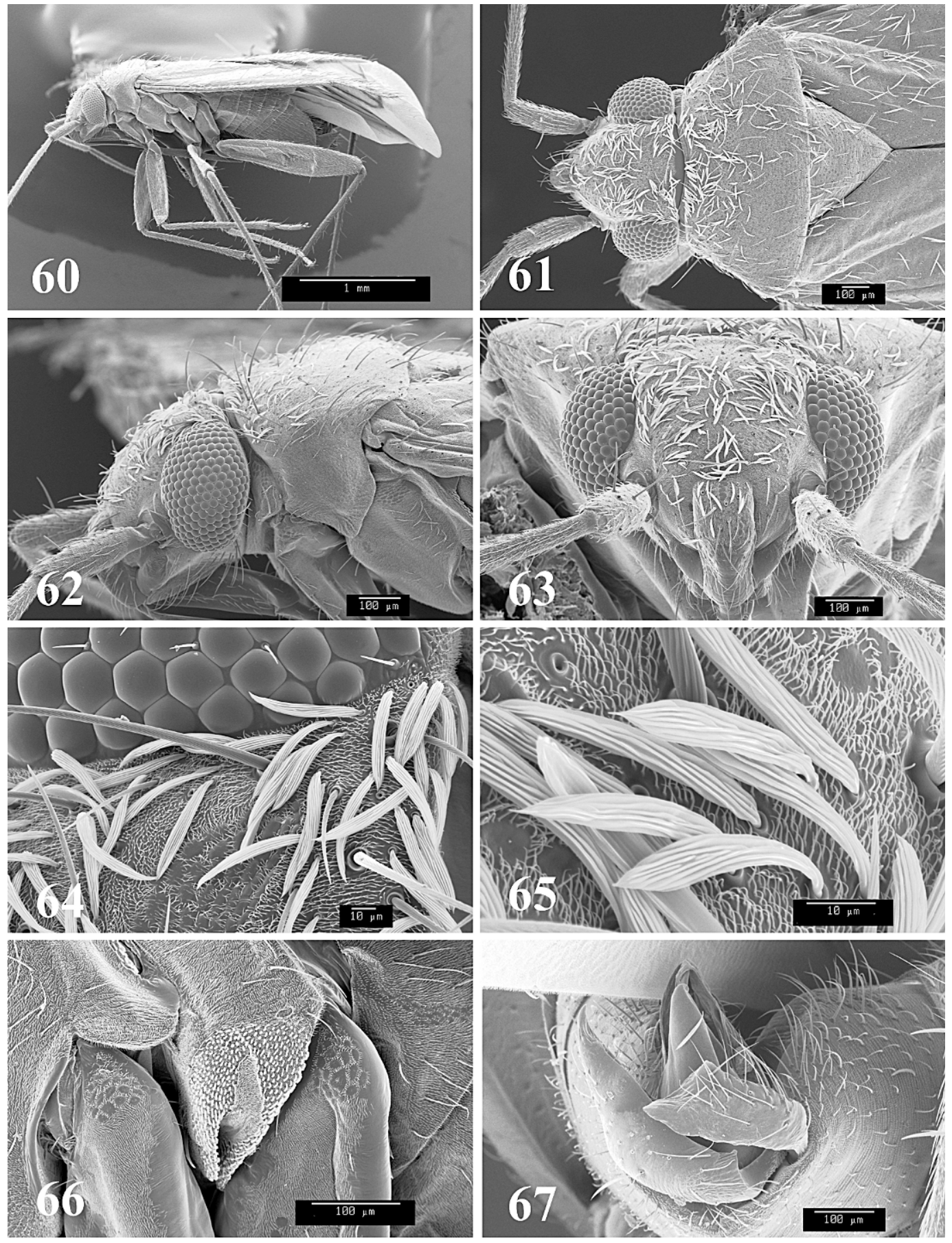

Figs. 60-67. Scanning electron photomicrographs of adult male Parthenicus wheeleri. 60, Lateral aspect $(30.0 \times)$. 61, Head and pronotum, dorsal aspect $(72.5 \times)$. 62. Head and pronotum, lateral aspect $(109 \times) .63$, Head, frontal aspect $(126 \times)$. 64, Scalelike setae on head $(635 \times)$. 65, Scalelike setae on head $(1800 \times)$. 66, Ostiolar evaporative area $(232 \times)$. 67, Genital capsule, caudal aspect $(156 \times)$. 

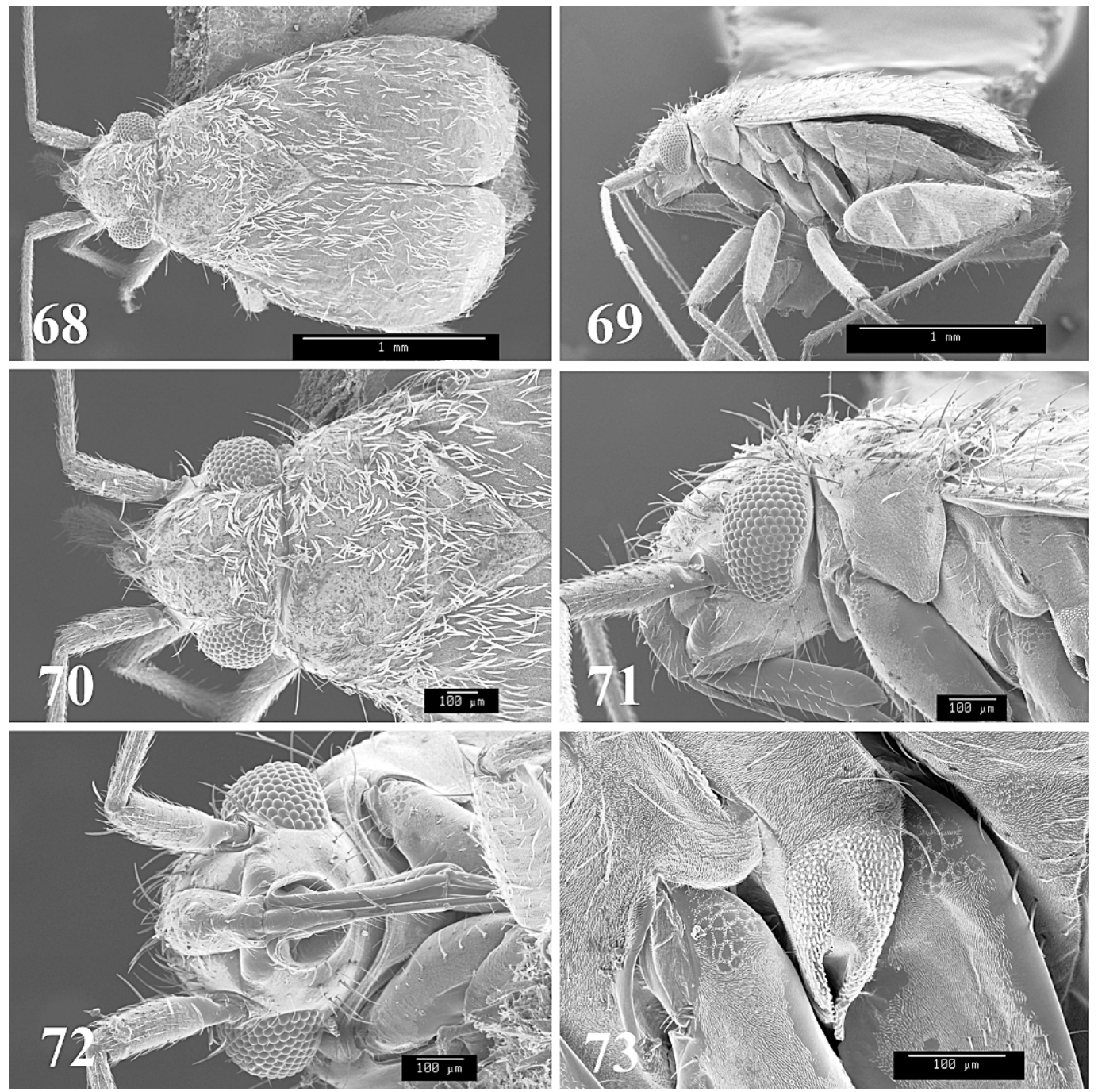

Figs. 68-73. Scanning electron photomicrographs of adult female Parthenicus wheeleri. 68, Dorsal aspect $(41.2 \times)$. 69, Lateral aspect $(38.8 \times)$. 70, Head and pronotum, dorsal aspect $(70.5 \times)$. 71, Head and pronotum, lateral aspect $(106 \times)$. 72, Head, ventral aspect $(107 \times)$. 73, Ostiolar evaporative area $(218 \times)$.

Co.: Rt. 77, $2.4 \mathrm{~km} \mathrm{~N}$ of Carter Co. line, $5.0 \mathrm{~km} \mathrm{~S}$ of Turner Falls, 22 Apr 2002, A.G. Wheeler, Jr., Schizachyrium scoparium Michx. (Poaceae), $1 \hat{\delta}$ (AMNH_PBI 00071149) (USNM). Texas: Blanco Co.: Rt. 71, 4.9 km SE of Spicewood, $30.43^{\circ} \mathrm{N} 98.13^{\circ} \mathrm{W}, 1$ May 2002 , A.G. Wheeler, Jr., Muhlenbergia lindheimeri A.S. Hitchc. (Poaceae), $1 \hat{0}$ (AMNH_PBI 00071150), 3 우 (AMNH_PBI 00071151-00071153) (USNM). Burnet Co.: Rt. $71,2 \mathrm{~km}$ SE of Spicewood, $30.458^{\circ} \mathrm{N} 98.153^{\circ} \mathrm{W}, 1$ May 2002, A.G. Wheeler, Jr., Muhlenbergia lindheimeri A.S. Hitchc. (Poaceae), 2 ( ${ }^{\circ} \mathrm{MNH}_{-}$
PBI 00071154,AMNH_PBI 00071155)(USNM). Callahan Co.: I-20 Frontage Road, ar CR-119, ca. $7 \mathrm{~km}$ E of Clyde, $32.405^{\circ} \mathrm{N} 99.418^{\circ} \mathrm{W}, 20$ May 2004, A.G. Wheeler, Jr., Eragrostis curvula Schrad. (Poaceae), 5§̊ (AMNH_PBI 0007118000071184), 5우 (AMNH_PBI 0007122000071224) (USNM). Gillespie Co.: Rt. 16, $15 \mathrm{~km}$ NE of Fredericksburg, $30.36^{\circ} \mathrm{N} 98.75^{\circ} \mathrm{W}$, 27 May 2001, A.G. Wheeler, Jr., Muhlenbergia lindheimeri A.S. Hitchc. (Poaceae), $1 \hat{\delta}$ (AMNH_PBI 00071156), 2 ㅇ (AMNH_PBI 00071157, AMNH_PBI 00071158) (USNM). 

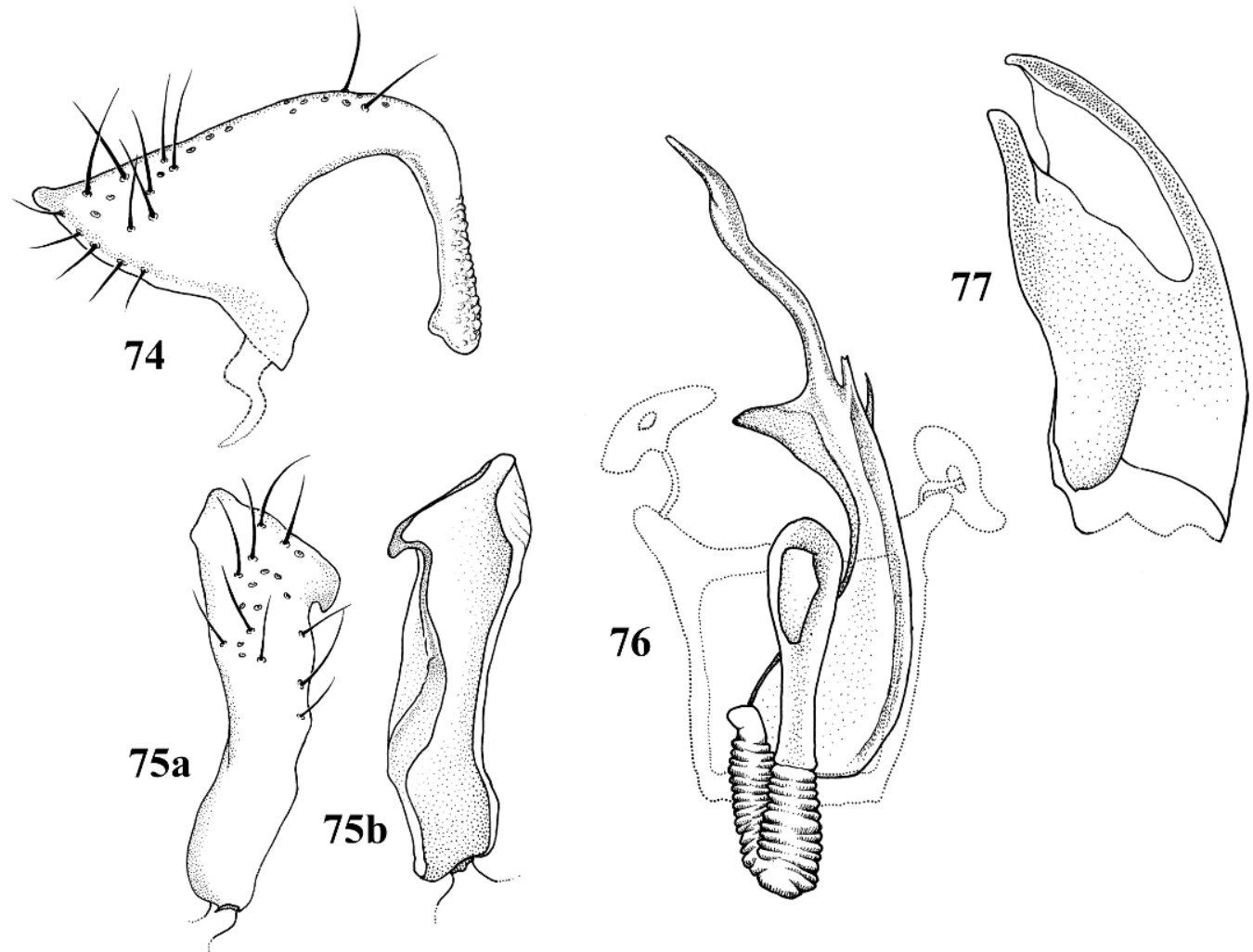

Figs. 74-77. Male genitalia of Parthenicus wheeleri. 74, Left paramere. 75, Right paramere (a, caudal aspect; b, anterior aspect). 76, Vesica. 77, Phallotheca.

Kerr Co.: Rt. $16,7.0 \mathrm{~km}$ NE of Kerrville, $30.09^{\circ} \mathrm{N} 99.08^{\circ} \mathrm{W}, 27$ May 2001, A.G. Wheeler, Jr., Muhlenbergia reverchonii Vasey \& Scribn. (Poaceae), $1 \hat{\delta}$ (AMNH_PBI 00071159) (USNM). Mills Co.: Farm Road 574, $5.0 \mathrm{~km} \mathrm{~W} \mathrm{of}$ Goldthwaite, $31.45^{\circ} \mathrm{N} 98.62^{\circ} \mathrm{W}, 26$ May 2001 , A.G. Wheeler, Jr., Schizachyrium scoparium Michx. (Poaceae), 12 ô (AMNH_PBI 0007116800071179), 24우 (AMNH_PBI 00071074, AMNH_PBI 00071197-00071219) (AMNH, CNC, USNM). Rt. 16, $9 \mathrm{~km}$ SW of Goldthwaite, $31.3977^{\circ} \mathrm{N} 98.6438^{\circ} \mathrm{W}, 30$ May 2001, A.G. Wheeler, Jr., Muhlenbergia lindheimeri A.S. Hitchc. (Poaceae), $8 \hat{\jmath}$ (AMNH PBI 00071160-00071167), 5ㅇ (AMNH_PBI 00071187-00071190, AMNH_PBI 00071196) (USNM); 2 May 2002, A.G. Wheeler, Jr., Schizachyrium scoparium Michx. (Poaceae), 4우 (AMNH_PBI 00071191-00071194) Muhlenbergia reverchonii Vasey \& Scribn. (Poaceae), 1 우 (AMNH_PBI 00071195) (USNM). Tarrant Co.:
Fort Worth Nature Center, Fort Worth, $32.84382^{\circ} \mathrm{N} 97.47879^{\circ} \mathrm{W}, 31$ May 2001, A.G. Wheeler, Jr., Schizachyrium scoparium Michx. (Poaceae), $1 \hat{\delta}$ (USNM). Travis Co.: Ranch W of Briarcliff, $30.4^{\circ} \mathrm{N} 98.057^{\circ} \mathrm{W}, 29$ May 2001, A.G. Wheeler, Jr., Muhlenbergia lindheimeri A.S. Hitchc. (Poaceae), $3+$ (USNM).

\section{ACKNOWLEDGMENTS}

I am grateful to Geoffroy Thomas (visiting scientific illustrator, Queensland Museum, Brisbane, Australia) for the color dorsal habitus illustration of $P$. sedumicola, Michele Touchet (Systematic Entomology Laboratory [SEL], ARS, USDA, c/o USNM) for the color photographic plates and database locality information, Katy Schuler (Washington, DC) for inking the genitalic drawings, and Alfred G. Wheeler, Jr. (Clemson University, 
Clemson, SC) for many of the specimens used in this study, including exclusive series of the new species $P$. wheeleri. I also thank Michael D. Schwartz (CNC) for providing specimen data and R.T. Schuh (AMNH) for lending specimens. John W. Brown (SEL), Alex S. Konstantinov (SEL), and Michael D. Schwartz (CNC) kindly reviewed the manuscript. This paper represents a contribution to NSF Planetary Biodiversity Inventory award DEB-0316495, R.T. Schuh, principal investigator (http://research.amnh.org/pbi/).

\section{REFERENCES}

Akingbohungbe, A.E., J.L. Libby, and R.D. Shenefelt. 1972. Miridae of Wisconsin (Hemiptera: Heteroptera). University of Wisconsin Research Bulletin R2396: 1-24.

Akingbohungbe, A.E., J.L. Libby, and R.D. Shenefelt. 1973. Nymphs of Wisconsin Miridae (Hemiptera: Heteroptera). University of Wisconsin Research Bulletin R2561: 1-25.

Atkinson, E.T. 1890. Catalogue of the Insecta. No. 2. Order Rhynchota. Suborder HemipteraHeteroptera. Family Capsidae. Journal of the Asiatic Society of Bengal 58(2): 25-200.

Barber, H.G. 1914. Insects of Florida. II. Hemiptera. Bulletin of the American Museum of Natural History 33: 495-535.

Blatchley, W.S. 1926. Heteroptera or true bugs of eastern North America, with especial reference to the faunas of Indiana and Florida. Indianapolis: Nature Publishing, 1116 pp.

Carvalho, J.C.M. 1952. On the major classification of the Miridae (Hemiptera). (With keys to subfamilies and tribes and a catalogue of the world genera). Anais da Academia Brasileira de Ciencias 24: 31-111.

Carvalho, J.C.M. 1958. Catalogue of the Miridae of the world. Part III. Orthotylinae. Arquivos do Museu Nacional, Rio de Janeiro 47: 1-161.

Froeschner, R.C. 1949. Contributions to a synopsis of the Hemiptera of Missouri, Pt. IV. Hebridae, Mesoveliidae, Cimicidae, Anthocoridae, Cryptostemmatidae, Isometopidae, Meridae [sic]. American Midland Naturalist 42: 123-188.

Heidemann, O. 1892. Note on the food-plants of some Capsidae from the vicinity of Washington, D.C. Proceedings of the Entomological Society of Washington 2: 224-226.

Heidemann, O. 1905. A list of capsids from the state of New York, with the description of a new species. Journal of the New York Entomological Society 13: 48-50.
Henry, T.J. 1978. Description of a new Polymerus, with notes on two other little known mirids from the New Jersey Pine-Barrens (Hemiptera: Miridae). Proceedings of the Entomological Society of Washington 80: 543-547.

Henry, T.J. 1982. Genus Parthenicus in the eastern United States, with descriptions of new species (Hemiptera: Miridae). Florida Entomologist 65: 354-366.

Henry, T.J. 2003. Proboscidotylus nigrosquamus (Maldonado) (Heteroptera: Miridae: Orthotylinae): new combination and first record for the United States. Proceedings of the Entomological Society of Washington 105: 59-65.

Henry, T.J., C.V. Covell, Jr., and A.G. Wheeler, Jr. 2005. An annotated list of the plant bugs, or Miridae (Hemiptera: Heteroptera), of Kentucky. Journal of the New York Entomological Society 113: 24-76.

Henry, T.J., and D.J. Hilburn. 1990. An annotated list of the true bugs (Heteroptera) of Bermuda. Proceedings of the Entomological Society of Washington 92: 675-684.

Henry, T.J., and C.L. Smith. 1979. An annotated list of the Miridae of Georgia (HemipteraHeteroptera). Journal of the Georgia Entomological Society 14: 212-220.

Henry, T.J., and A.G. Wheeler, Jr. 1988. Family Miridae Hahn, 1833 (= Capsidae Burmeister, 1835). In T.J. Henry and R.C. Froeschner (editors), Catalog of the Heteroptera, or true bugs, of Canada and the eastern United States: 251-507. Leiden and New York: E. J. Brill, $958 \mathrm{pp}$.

Kirkaldy, G.W. 1906. List of the genera of the pagiopodus Hemiptera-Heteroptera, with their types species, from 1758 to 1904 and also of the aquatic and semi-aquatic Trocholaopoda. Transactions of the American Entomological Society 32: 117-156.

Knight, H.H. 1918. Synoptic key to the subfamilies of Miridae (Hemiptera-Heteroptera). Journal of the New York Entomological Society 26: $40-44$.

Knight, H.H. 1919. Interesting new species of Miridae from the United States, with a note on Orthocephalus mutabilis (Fallen) (Hemip. Miridae). Bulletin of the Brooklyn Entomological Society 13: 111-116 (1918).

Knight, H.H. 1923. Family Miridae (Capsidae). In W.E. Britton (editor), Hemiptera of Connecticut. Connecticut State Geological and Natural History Survey Bulletin 22: 422-655.

Knight, H.H. 1941. The plant bugs, or Miridae, of Illinois. Illinois Natural History Survey Bulletin 22. 234 pp.

Knight, H.H. 1968. Taxonomic review: Miridae of the Nevada test site and the western United 
States. Brigham Young University Science Bulletin 9(3): 1-282.

Maw, H.E.L., R.G. Foottit, K.G.A. Hamilton, and G.G.E. Scudder. 2000. Checklist of the Hemiptera of Canada and Alaska. Ottawa: NRC Research Press, 220 pp.

Reuter, O.M. 1876. Capsinae ex America boreali in Museo Homiensi asservatae, descriptae. Öfversigt Kongliga Svenska VetenskapsAkademiens Förhandlingar 32(9): 59-92.

Roble, S.M., and R.L. Hoffman. 2000. Three true bugs new to the Virginia fauna, including the first record of the family Schizopteridae (Heteroptera). Banisteria 16: 41-45.

Schuh, R.T. 1974. The Orthotylinae and Phylinae (Hemiptera: Miridae) of South Africa with a phylogenetic analysis of the ant-mimetic tribes of the two subfamilies for the world. Entomologica Americana 47: 1-332.

Schuh, R.T. 1995. Plant bugs of the world (Insecta: Heteroptera: Miridae). Systematic catalog, distributions, host list, and bibliography. New York: New York Entomological Society, 1329 pp. [see also updated online version: http://research.amnh.org/pbi/catalog].

Schwartz, M.D., and G.G.E. Scudder. 2003. Seven new species of Miridae (Heteroptera) from British Columbia and Alaska and synonymy of Adelphocoris superbus (Uhler). Journal of the New York Entomological Society 111: 65-95.

Snograss, G.L., T.J. Henry, and W.P. Scott. 1984. An annotated list of the Miridae (Heteroptera) found in the Yazoo-Mississippi Delta and associated areas in Arkansas and Louisiana. Proceedings of the Entomological Society of Washington 86: 845-860.

Uhler, P.R. 1886. Check-list of the Hemiptera Heteroptera of North America. New York: Brooklyn Entomological Society, 32 pp.
USDA, NRCS. 2004. The PLANTS database. Version 3.5 (http://plants.usda.gov.): National Plant Data Center: Baton Rouge, LA. [accessed October 2005]

Van Duzee, E.P. 1915. New genera and species of North American Hemiptera. Pomona Journal of Entomology and Zoology 7: 109-121.

Van Duzee, E.P. 1916. Checklist of the Hemiptera (excepting the Aphididae, Aleurodidae and Coccidae) of America north of Mexico. New York: New York Entomological Society, $111 \mathrm{pp}$.

Van Duzee, E.P. 1917. Catalogue of the Hemiptera of America north of Mexico excepting the Aphididae, Coccidae and Aleurodidae. Berkeley: University of California Press, 902 pp.

Van Duzee, E.P. 1918. New species of Hemiptera chiefly from California. Proceedings of the California Academy of Sciences (4)8: 271-308.

Van Duzee, E.P. 1923. Expedition of the California Academy of Sciences to the Gulf of California in 1921. Proceedings of the California Academy of Sciences (4)12: 123-200.

Wheeler, A.G., Jr., and T.J. Henry. 1975. Recognition of seven Uhler manuscript names, with notes on thirteen other species used by Heidemann (1892) (Hemiptera: Miridae). Transactions of the American Entomological Society 101: 355-369.

Wheeler, A.G., Jr., and T.J. Henry. 1977. Miridae associated with Pennsylvania conifers 1 . Species on arborvitae, false cypress, and juniper. Transactions of the American Entomological Society 103: 623-656.

Wheeler, A.G., Jr., T.J. Henry, and T.L. Mason, Jr. 1983. An annotated list of the Miridae of West Virginia (Hemiptera-Heteroptera). Transactions of the American Entomological Society 109: $127-159$. 

Complete lists of all issues of the Novitates and the Bulletin are available at World Wide Web site http://library.amnh.org/pubs. Inquire about ordering printed copies via e-mail from scipubs@amnh.org or via standard mail from: American Museum of Natural History, Library-Scientific Publications, Central Park West at 79th St., New York, NY 10024. TEL: (212) 769-5545. FAX: (212) 769-5009. 